\title{
A NEW FORMULATION OF THE STOKES PROBLEM IN A CYLINDER, AND ITS SPECTRAL DISCRETIZATION
}

\author{
Nehla AbDellatif ${ }^{1}$ And Christine Bernardi ${ }^{2}$
}

\begin{abstract}
We analyze a new formulation of the Stokes equations in three-dimensional axisymmetric geometries, relying on Fourier expansion with respect to the angular variable: the problem for each Fourier coefficient is two-dimensional and has six scalar unknowns, corresponding to the vector potential and the vorticity. A spectral discretization is built on this formulation, which leads to an exactly divergence-free discrete velocity. We prove optimal error estimates.
\end{abstract}

Mathematics Subject Classification. 65N35.

Received: May 16, 2003.

\section{INTRODUCTION}

The Stokes equations govern the flow of a viscous incompressible fluid in the case of very small velocities, indeed their primitive variables are the velocity and the pressure of the fluid. The aim of this paper is to describe and analyze a new spectral type discretization of these equations in a cylinder, that relies on two ideas:

- Thanks to the axisymmetry of the domain, the use of truncated Fourier series allows for computing a discrete three-dimensional solution by only solving a few number of discrete problems on the meridian domain.

- For each problem set in the meridian domain, the vector potential and vorticity can be used as new unknowns.

Indeed, on one hand, in the special case of three-dimensional axisymmetric geometries, it is proven in ([10], Chap. I) that, thanks to a Fourier expansion with respect to the angular variable, these equations reduce to an infinite family of uncoupled problems set in the two-dimensional meridian domain. The corresponding variational formulation of each two-dimensional problem, which involves weighted Sobolev spaces, and its well-posedness are also presented in ([10] Sect. IX.1), together with its spectral discretization. Moreover, the Spectral - Fourier discretization of the three-dimensional problem relies on Fourier truncation and consists in approximating only a finite number of two-dimensional problems by spectral techniques: its numerical analysis is performed in ([10], Chap. X) and leads to error estimates of spectral type, i.e. the order of the error only depends on the regularity of the exact solution. The idea for using spectral techniques rather than finite elements is that they are well appropriate for being coupled with Fourier truncation since they have the same infinite degree of accuracy.

Keywords and phrases. Stokes problem, spectral methods, axisymmetric geometries.

1 École Nationale des Sciences de l'Informatique, Campus Universitaire, 2010 Manouba, Tunisia.

2 Laboratoire Jacques-Louis Lions, CNRS \& Université Pierre et Marie Curie, B.C. 187, 4 place Jussieu, 75252 Paris Cedex 05, France. e-mail: bernardi@ann.jussieu.fr 
On the other hand, a well-known method for the discretization of the Stokes problem in two-dimensional domains, first analyzed in [14] in the finite element framework and extended in [8] to spectral type discretizations, relies on the stream function and vorticity formulation: indeed, the incompressibility equation is equivalent to the fact that the velocity is the curl of this stream function and the curl of the velocity, which is the vorticity, can be used as a second unknown. This results into a system of two Laplace equations which are coupled by the boundary conditions on the stream function. This technique presents two main advantages: it is not expensive since only two scalar unknowns are involved in the resulting formulation and it leads to exactly divergence-free velocities which is important for instance when convection equations are coupled with the Stokes problem.

So, the idea of this paper consists in extending to the two-dimensional systems resulting from the reduction of the three-dimensional Stokes problem in a cylinder the stream function and vorticity technique. This has already been performed by Abdellatif, see [1,2], in the case of axisymmetric data, i.e. for the Fourier coefficient of order zero: in this case, the Stokes system results into a Laplace equation for the angular component of the velocity and another problem for the three other unknowns which can be formulated with a scalar stream function and vorticity as only unknowns. We refer to [2] for a detailed analysis of the corresponding variational formulation and spectral discretization, we only recall these results. However, for other Fourier coefficients, the four equations of the Stokes reduced system are coupled: We propose here to choose as unknowns the vector potential and the vorticity related to the velocity. Note however that, in this case, an additional gauge condition must be enforced on the vector potential in order to ensure its uniqueness.

In general three-dimensional geometries, the Stokes problem with these new unknowns results into a system of two coupled second-order vectorial equations. Several variational formulations of these problems exist, we choose to use one which has been recently proposed and studied by Amara, Barucq and Duloué [4] (see [3] for a first announcement and [13] for complementary results): a further decomposition of the unknowns is added, which is linked to the well-known Glowinski and Pironneau algorithm [16] and seems very efficient for solving the linear system resulting from the discretization.

We first write the formulation proposed in [4] in the case of an axisymmetric domain: it results into a countable system of uncoupled two-dimensional variational problems, one for each Fourier coefficient. We prove its well-posedness in the appropriate weighted Sobolev spaces. Next, we describe the spectral discretization of the problem satisfied by each Fourier coefficient and we perform its numerical analysis, in the case of a model cylinder. Note however that the discretization can be extended to more general axisymmetric geometries, by transformation and decomposition of the domain, relying on the spectral element method. We also describe the Spectral - Fourier discretization in this case, obtained by combining the previous discretization with Fourier truncature, and give the corresponding three-dimensional error estimates.

The extension of this discretization to the full nonlinear Navier-Stokes equations, is presently under consideration. We also intend to handle more complex axisymmetric geometries by the spectral element method.

An outline of the paper is as follows:

- In Section 2, we briefly recall the variational formulations and the well-posedness results of [4] for general three-dimensional geometries.

- In Section 3, we present the variational formulations of the two problems satisfied by each Fourier coefficient of the vector potential and vorticity, and we check that they are well-posed. We also estimate the error issued from Fourier truncature.

- In Section 4, we describe the corresponding discrete problems, we prove their well-posedness and we establish optimal error estimates.

- Some results which require technical arguments are proved in Appendices A and B.

\section{The THREE-DIMENSIONAL VECTOR POTENTIAL AND VORTICITY FORMULATION}

Let $\breve{\Omega}$ be a three-dimensional bounded connected domain with a Lipschitz-continuous boundary $\partial \breve{\Omega}$ and generic point $\breve{\boldsymbol{x}}=(x, y, z)$. For simplicity, we assume from now on that this domain has a connected boundary and is simply-connected (more general geometries can be handled from the analysis given e.g. in [5]). We denote 
by $\breve{\boldsymbol{n}}$ the unit outward normal to $\breve{\Omega}$ on $\partial \breve{\Omega}$. The Stokes problem in this domain and in Cartesian coordinates reads:

$$
\begin{cases}-\Delta \breve{\boldsymbol{u}}+\operatorname{grad} \breve{p}=\breve{\boldsymbol{f}} & \text { in } \breve{\Omega}, \\ \operatorname{div} \breve{\boldsymbol{u}}=0 & \text { in } \breve{\Omega}, \\ \breve{\boldsymbol{u}}=\mathbf{0} & \text { on } \partial \breve{\Omega},\end{cases}
$$

where the unknowns are the velocity $\breve{\boldsymbol{u}}$ and the pressure $\breve{p}$. The data are a density of body forces $\breve{f}$ and, only for simplicity, we take homogeneous boundary conditions on the velocity. It is well-known that, for any data $\breve{f}$ in the dual space $H^{-1}(\breve{\Omega})^{3}$ of $H_{0}^{1}(\breve{\Omega})^{3}$, this problem admits a variational solution $(\breve{\boldsymbol{u}}, \breve{p})$ in $H_{0}^{1}(\breve{\Omega})^{3} \times L^{2}(\breve{\Omega})$, which is unique up to an additive constant on the pressure.

We now introduce the new unknowns. The first one is the vorticity $\breve{\omega}$ defined by

$$
\breve{\omega}=\operatorname{curl} \breve{\boldsymbol{u}} \quad \text { in } \breve{\Omega} .
$$

As far as the second one is concerned, we recall ([5], Th. 3.17) that, since $\breve{\boldsymbol{u}}$ is divergence-free in $\breve{\Omega}$ and has its normal component equal to zero on $\partial \breve{\Omega}$, there exists a unique vector potential $\breve{\psi}$ such that

$$
\begin{cases}\breve{\boldsymbol{u}}=\operatorname{curl} \breve{\boldsymbol{\psi}} & \text { in } \breve{\Omega}, \\ \operatorname{div} \breve{\boldsymbol{\psi}}=0 & \text { in } \breve{\Omega} \\ \breve{\boldsymbol{\psi}} \times \breve{\boldsymbol{n}}=\mathbf{0} & \text { on } \partial \breve{\Omega}\end{cases}
$$

This leads to the modified equivalent formulation of the Stokes problem

$$
\begin{cases}\operatorname{curl} \operatorname{curl} \breve{\boldsymbol{\omega}}=\operatorname{curl} \breve{\boldsymbol{f}} & \text { in } \breve{\Omega}, \\ \operatorname{curl} \operatorname{curl} \breve{\boldsymbol{\psi}}=\breve{\boldsymbol{\omega}} & \text { in } \breve{\Omega}, \\ \operatorname{div} \breve{\boldsymbol{\psi}}=\operatorname{div} \breve{\boldsymbol{\omega}}=0 & \text { in } \breve{\Omega}, \\ \breve{\boldsymbol{\psi}} \times \breve{\boldsymbol{n}}=\operatorname{curl} \breve{\boldsymbol{\psi}} \times \breve{\boldsymbol{n}}=\mathbf{0} & \text { on } \partial \breve{\Omega}\end{cases}
$$

However, following [3,4], we consider a further decomposition of the vorticity which is used for the Glowinski and Pironneau algorithm [16] in the two-dimensional case. We set: $\breve{\boldsymbol{\omega}}=\breve{\boldsymbol{\omega}}^{0}+\breve{\boldsymbol{\omega}}^{*}$, so that the previous problem (2.4) can be written as a system of two uncoupled problems:

$$
\begin{cases}\operatorname{curl} \operatorname{curl} \breve{\boldsymbol{\omega}}^{0}=\operatorname{curl} \breve{\boldsymbol{f}} & \text { in } \breve{\Omega}, \\ \operatorname{div} \breve{\boldsymbol{\omega}}^{0}=0 & \text { in } \breve{\Omega}, \\ \breve{\boldsymbol{\omega}}^{0} \times \breve{\boldsymbol{n}}=\mathbf{0} & \text { on } \partial \breve{\Omega}\end{cases}
$$

and

$$
\begin{cases}\operatorname{curl} \operatorname{curl} \breve{\boldsymbol{\omega}}^{*}=\mathbf{0} & \text { in } \breve{\Omega}, \\ \operatorname{curl} \operatorname{curl} \breve{\boldsymbol{\psi}}=\breve{\boldsymbol{\omega}}^{0}+\breve{\boldsymbol{\omega}}^{*} & \text { in } \breve{\Omega}, \\ \operatorname{div} \breve{\boldsymbol{\psi}}=\operatorname{div} \breve{\boldsymbol{\omega}}^{*}=0 & \text { in } \breve{\Omega}, \\ \breve{\boldsymbol{\psi}} \times \breve{\boldsymbol{n}}=\operatorname{curl} \breve{\boldsymbol{\psi}} \times \breve{\boldsymbol{n}}=\mathbf{0} & \text { on } \partial \breve{\Omega}\end{cases}
$$

The variational formulation of these problems involves the space $H$ (curl, $\breve{\Omega})$ of vector fields in $L^{2}(\breve{\Omega})^{3}$ such that their curl belong to $L^{2}(\breve{\Omega})^{3}$. Relying on the standard result ([15], Chap. I, Th. 2.11) that the trace 
mapping: $\breve{\boldsymbol{v}} \mapsto \breve{\boldsymbol{v}} \times \breve{\boldsymbol{n}}$ is continuous from $H(\mathbf{c u r l}, \breve{\Omega})$ into $H^{-\frac{1}{2}}(\partial \breve{\Omega})^{3}$ (defined as the dual space of $\left.H^{\frac{1}{2}}(\partial \breve{\Omega})^{3}\right)$, we define its closed subspace

$$
X(\breve{\Omega})=\{\breve{\boldsymbol{v}} \in H(\operatorname{curl}, \breve{\Omega}) ; \breve{\boldsymbol{v}} \times \breve{\boldsymbol{n}}=\mathbf{0} \text { on } \partial \breve{\Omega}\}
$$

Note also ([4], Prop. 2.1) that the mapping: $\breve{\boldsymbol{v}} \mapsto \operatorname{curl} \breve{\boldsymbol{v}}$ is continuous from $L^{2}(\breve{\Omega})^{3}$ into the dual space $X(\breve{\Omega})^{\prime}$ of $X(\breve{\Omega})$. We finally introduce the space

$$
Y(\breve{\Omega})=\left\{\breve{\boldsymbol{v}} \in L^{2}(\breve{\Omega})^{3} ; \operatorname{curl} \operatorname{curl} \breve{\boldsymbol{v}} \in X(\breve{\Omega})^{\prime}\right\} .
$$

From now on, we assume that the data $\breve{f}$ belong to $L^{2}(\breve{\Omega})^{3}$. The variational formulation of problem $(2.5)$ is of saddle-point type, it reads:

$$
\begin{aligned}
& \text { Find } \breve{\boldsymbol{\omega}}^{0} \text { in } X(\breve{\Omega}) \text { and } \breve{\lambda}^{0} \text { in } H_{0}^{1}(\breve{\Omega}) \text { such that } \\
& \forall \breve{\varphi} \in X(\breve{\Omega}), \int_{\breve{\Omega}} \operatorname{curl} \breve{\omega}^{0} \cdot \operatorname{curl} \breve{\varphi} \mathrm{d} \breve{\boldsymbol{x}}+\int_{\breve{\Omega}} \operatorname{grad} \breve{\lambda}^{0} \cdot \breve{\varphi} \mathrm{d} \breve{\boldsymbol{x}}=\int_{\breve{\Omega}} \breve{f} \cdot \operatorname{curl} \breve{\varphi} \mathrm{d} \breve{\boldsymbol{x}}, \\
& \forall \breve{\mu} \in H_{0}^{1}(\breve{\Omega}), \int_{\breve{\Omega}} \operatorname{grad} \breve{\mu} \cdot \breve{\omega}^{0} \mathrm{~d} \breve{\boldsymbol{x}}=0 .
\end{aligned}
$$

The variational formulation of problem (2.6) is still of saddle-point type, however it is slightly more complicated. It reads:

$$
\begin{aligned}
& \text { Find }\left(\breve{\boldsymbol{\omega}}^{*}, \breve{\lambda}^{*}\right) \text { in } Y(\breve{\Omega}) \times H_{0}^{1}(\breve{\Omega}) \text { and } \breve{\boldsymbol{\psi}} \text { in } X(\breve{\Omega}) \text { such that } \\
& \forall(\breve{\boldsymbol{\vartheta}}, \breve{\mu}) \in Y(\breve{\Omega}) \times H_{0}^{1}(\breve{\Omega}), \\
& \quad \int_{\breve{\Omega}} \breve{\boldsymbol{\omega}}^{*} \cdot \breve{\boldsymbol{\vartheta}} \mathrm{d} \breve{\boldsymbol{x}}-\langle\operatorname{curl} \operatorname{curl} \breve{\boldsymbol{\vartheta}}, \breve{\boldsymbol{\psi}}\rangle+\int_{\breve{\Omega}} \breve{\boldsymbol{\psi}} \cdot \operatorname{grad} \breve{\mu} \mathrm{d} \breve{\boldsymbol{x}}=-\int_{\breve{\Omega}} \breve{\boldsymbol{\omega}}^{0} \cdot \breve{\boldsymbol{\vartheta}} \mathrm{d} \breve{\boldsymbol{x}}, \\
& \forall \breve{\boldsymbol{\varphi}} \in X(\breve{\Omega}), \quad-\left\langle\operatorname{curl} \operatorname{curl} \breve{\boldsymbol{\omega}}^{*}, \breve{\boldsymbol{\varphi}}\right\rangle+\int_{\breve{\Omega}} \breve{\boldsymbol{\varphi}} \cdot \operatorname{grad} \breve{\lambda}^{*} \mathrm{~d} \breve{\boldsymbol{x}}=0,
\end{aligned}
$$

where $\langle\cdot, \cdot\rangle$ stands for the duality pairing between $X(\breve{\Omega})^{\prime}$ and $X(\breve{\Omega})$. It can be observed that problem $(2.9)$ is completely independent of problem (2.10), so that they can be solved separately.

We conclude this section by recalling the main results concerning these problems, which are proven in ([4], Thms. 3.1, 3.2 and 4.1):

(i) Problem (2.9) admits a unique solution $\left(\breve{\boldsymbol{\omega}}^{0}, \breve{\lambda}^{0}\right)$ in $X(\breve{\Omega}) \times H_{0}^{1}(\breve{\Omega})$, with $\breve{\lambda}^{0}=0$, and is equivalent to problem (2.5).

(ii) Problem $(2.10)$ admits a unique solution $\left(\breve{\boldsymbol{\omega}}^{*}, \breve{\lambda}^{*}, \breve{\boldsymbol{\psi}}\right)$ in $Y(\breve{\Omega}) \times H_{0}^{1}(\breve{\Omega}) \times X(\breve{\Omega})$, with $\breve{\lambda}^{*}=0$, and is equivalent to problem (2.6).

(iii) If the function $\breve{\boldsymbol{u}}$ is defined by $\breve{\boldsymbol{u}}=\operatorname{curl} \breve{\boldsymbol{\psi}}$, where $\left(\breve{\boldsymbol{\omega}}^{*}, \breve{\lambda}^{*}, \breve{\boldsymbol{\psi}}\right)$ is the solution of problem (2.10), there exists a function $\breve{p}$, unique up to an additive constant, such that the pair $(\breve{\boldsymbol{u}}, \breve{p})$ belongs to $H_{0}^{1}(\breve{\Omega})^{3} \times L^{2}(\breve{\Omega})$ and is the unique solution of problem (2.1).

So, from now on, we are interested with problems (2.9) and (2.10) in the special case of an axisymmetric geometry, namely of a cylinder.

Remark. Assume that $\breve{\Omega}$ is a cylinder and also that the data $\breve{f}$ belongs to $H^{2}(\breve{\Omega})^{3}$. The regularity properties of the Stokes problem, as stated in ([10], Sect. IX.1.b) yield the properties

$$
\operatorname{curl} \breve{\psi} \in H^{3.739}(\breve{\Omega})^{3}, \quad \breve{\omega} \in H^{2.739}(\breve{\Omega})^{3} .
$$


However the separate regularity of $\breve{\omega}^{0}$ and $\breve{\omega}^{*}$ is weaker. By using the arguments in [12] and the fact that the singularities of the Laplace equation in a cylinder are explicitly known (see [10], Th. II.4.9) we derive that, for all positive $\varepsilon$,

$$
\breve{\boldsymbol{\omega}}^{0} \in H^{2-\varepsilon}(\breve{\Omega})^{3}, \quad \operatorname{curl} \breve{\boldsymbol{\omega}}^{0} \in H^{2-\varepsilon}(\breve{\Omega})^{3} .
$$

The same argument, combined with (2.11), finally yields

$$
\breve{\boldsymbol{\omega}}^{*} \in H^{2-\varepsilon}(\breve{\Omega})^{3}, \quad \operatorname{curl} \breve{\omega}^{*} \in H^{2-\varepsilon}(\breve{\Omega})^{3}, \quad \breve{\psi} \in H^{2-\varepsilon}(\breve{\Omega})^{3}
$$

Another type of regularity results, concerning only the dependence with the angular variable in axisymmetric domains, is stated later on.

\section{THE TWO-DIMENSIONAL REDUCED FORMULATION FOR AXISYMMETRIC DOMAINS}

Let $\Omega$ be a bounded open polygon in the $(r, z)$ half-plane $] 0,+\infty\left[\times \mathbb{R}\right.$. We denote by $\Gamma_{0}$ the interior of the part of the boundary $\partial \Omega$ which is contained in the rotation axis $\{r=0\}$ and we set: $\Gamma=\partial \Omega \backslash \Gamma_{0}$. Let also $\boldsymbol{n}$ stand for the unit outward normal vector to $\Omega$ on $\Gamma$.

We now work in the three-dimensional domain $\breve{\Omega}$ which is built by rotating $\Omega \cup \Gamma_{0}$ around the axis $\{r=0\}$ :

$$
\breve{\Omega}=\left\{(r, \theta, z) ;(r, z) \in \Omega \cup \Gamma_{0} \text { and }-\pi \leq \theta<\pi\right\} .
$$

We assume that $\Gamma_{0}$ is not empty and is a finite union of disjoint segments (i.e. does not contain isolated points) and also that $\Omega$ is connected and simply-connected. We need some further notation.

Notation. With each scalar function $\breve{\mu}$ of the Cartesian variables $x, y$ and $z$, we associate the corresponding function $\mu$ of the cylindrical variables $r, z$ and $\theta$. For each vector field $\breve{\boldsymbol{v}}$ of the Cartesian variables $x, y$ and $z$, we agree to denote by $v_{r}, v_{\theta}$ and $v_{z}$ its radial, angular and axial components, respectively, which are functions of $r, \theta$ and $z$, and by $\boldsymbol{v}$ the vector with components $v_{r}, v_{\theta}$ and $v_{z}$.

With this notation, we recall that the curl and div operators of a function $\boldsymbol{v}$ in cylindrical variables and components, denoted by $\operatorname{curl}_{r}$ and $\operatorname{div}_{r}$ to avoid confusion, are given by

$$
\begin{aligned}
& \left(\operatorname{curl}_{r} \boldsymbol{v}\right)_{r}=r^{-1} \partial_{\theta} v_{z}-\partial_{z} v_{\theta}, \quad\left(\operatorname{curl}_{r} \boldsymbol{v}\right)_{\theta}=\partial_{z} v_{r}-\partial_{r} v_{z} \\
& \left(\operatorname{curl}_{r} \boldsymbol{v}\right)_{z}=\partial_{r} v_{\theta}+r^{-1} v_{\theta}-r^{-1} \partial_{\theta} v_{r} \\
& \operatorname{div}_{r} \boldsymbol{v}=\partial_{r} v_{r}+r^{-1} v_{r}+r^{-1} \partial_{\theta} v_{\theta}+\partial_{z} v_{z}
\end{aligned}
$$

Finally, we choose to use the Fourier development of all scalar functions and vector fields with respect to the angular variable $\theta$. For instance, for a scalar function $\mu$, this development writes

$$
\mu(r, \theta, z)=\frac{1}{\sqrt{2 \pi}} \sum_{k \in \mathbb{Z}} \mu^{k}(r, z) \mathrm{e}^{i k \theta}, \quad \text { with } \quad \mu^{k}(r, z)=\frac{1}{\sqrt{2 \pi}} \int_{-\pi}^{\pi} \mu(r, \theta, z) \mathrm{e}^{-i k \theta} \mathrm{d} \theta
$$

Thanks to this change of variables, problems (2.5) and (2.6) now result into a countable set of two-dimensional problems, namely, for any $k$ in $\mathbb{Z}$,

$$
\begin{cases}\operatorname{curl}_{k} \operatorname{curl}_{k} \boldsymbol{\omega}^{0 k}=\operatorname{curl}_{k} \boldsymbol{f}^{k} & \text { in } \Omega, \\ \operatorname{div}_{k} \boldsymbol{\omega}^{0 k}=0 & \text { in } \Omega, \\ \gamma_{T} \boldsymbol{\omega}^{0 k}=\mathbf{0} & \text { on } \Gamma,\end{cases}
$$


and

$$
\begin{cases}\operatorname{curl}_{k} \operatorname{curl}_{k} \boldsymbol{\omega}^{* k}=\mathbf{0} & \text { in } \Omega, \\ \operatorname{curl}_{k} \operatorname{curl}_{k} \boldsymbol{\psi}^{k}=\boldsymbol{\omega}^{0 k}+\boldsymbol{\omega}^{* k} & \text { in } \Omega, \\ \operatorname{div}_{k} \boldsymbol{\psi}^{k}=\operatorname{div}_{k} \boldsymbol{\omega}^{* k}=0 & \text { in } \Omega, \\ \gamma_{T} \boldsymbol{\psi}^{k}=\gamma_{T}\left(\mathbf{c u r l}_{k} \boldsymbol{\psi}^{k}\right)=\mathbf{0} & \text { on } \Gamma,\end{cases}
$$

where the operators $\operatorname{curl}_{k}$ and $\operatorname{div}_{k}$ are now given by

$$
\begin{aligned}
& \left(\operatorname{curl}_{k} \boldsymbol{v}\right)_{r}=i k r^{-1} v_{z}-\partial_{z} v_{\theta}, \quad\left(\operatorname{curl}_{k} \boldsymbol{v}\right)_{\theta}=\partial_{z} v_{r}-\partial_{r} v_{\boldsymbol{z}} \\
& \left(\operatorname{curl}_{k} \boldsymbol{v}\right)_{z}=\partial_{r} v_{\theta}+r^{-1} v_{\theta}-i k r^{-1} v_{r} \\
& \operatorname{div}_{k} \boldsymbol{v}=\partial_{r} v_{r}+r^{-1} v_{r}+i k r^{-1} v_{\theta}+\partial_{z} v_{z}
\end{aligned}
$$

and the tangential trace operator $\gamma_{T}$ is defined by

$$
\left(\gamma_{T} \boldsymbol{v}\right)_{r}=v_{\theta} n_{z}, \quad\left(\gamma_{T} \boldsymbol{v}\right)_{\theta}=v_{z} n_{r}-v_{r} n_{z}, \quad\left(\gamma_{T} \boldsymbol{v}\right)_{z}=-v_{\theta} n_{r}
$$

For completeness, we also introduce the operator $\operatorname{grad}_{k}$ on scalar functions $\mu$ by

$$
\left(\operatorname{grad}_{k} \mu\right)_{r}=\partial_{r} \mu, \quad\left(\operatorname{grad}_{k} \mu\right)_{\theta}=i k r^{-1} \mu, \quad\left(\operatorname{grad}_{k} \mu\right)_{z}=\partial_{z} \mu .
$$

We now describe the weighted Sobolev spaces which are needed for the variational formulations of these problems, next we write these formulations and we prove their well-posedness.

\subsection{The weighted Sobolev spaces}

Note that going from Cartesian variables $x, y$ and $z$ to cylindrical variables $r, \theta$ and $z$ transforms the Lebesgue measure into the weighted one $r \mathrm{~d} r \mathrm{~d} \theta \mathrm{d} z$. So, this change of variables leads to work with weighted Sobolev spaces on $\Omega$.

We first introduce the spaces:

$$
L_{ \pm 1}^{2}(\Omega)=\left\{v: \Omega \rightarrow \mathbb{R} \text { measurable; } \int_{\Omega}|v(r, z)|^{2} r^{ \pm 1} \mathrm{~d} r \mathrm{~d} z<+\infty\right\} .
$$

Next, we define the complete scale of Sobolev spaces $H_{1}^{s}(\Omega)$ :

- when $s$ is an integer, $H_{1}^{s}(\Omega)$ is the space of functions in $L_{1}^{2}(\Omega)$ such that all their partial derivatives of order $\leq s$ belong to $L_{1}^{2}(\Omega)$;

- when $s$ is not an integer, $H_{1}^{s}(\Omega)$ is defined by Hilbertian interpolation between $H_{1}^{[s]+1}(\Omega)$ and $H_{1}^{[s]}(\Omega)$, where $[s]$ stands for the integral part of $s$.

We also need the space of "flat" functions

$$
V_{1}^{1}(\Omega)=H_{1}^{1}(\Omega) \cap L_{-1}^{2}(\Omega) .
$$

All these spaces are provided with the norms and seminorms that result from their definitions. Due to the Fourier development, we also use the same spaces for functions from $\Omega$ into $\mathbb{C}$, we keep the same notation for simplicity. We finally define the spaces

$$
H_{1 \diamond}^{1}(\Omega)=\left\{v \in H_{1}^{1}(\Omega) ; v=0 \text { on } \Gamma\right\}, \quad V_{1 \diamond}^{1}(\Omega)=V_{1}^{1}(\Omega) \cap H_{1 \diamond}^{1}(\Omega) .
$$

We introduce the spaces, for any $k \in \mathbb{Z}$,

$$
H_{(k)}^{1}(\Omega)= \begin{cases}H_{1}^{1}(\Omega) & \text { if } k=0 \\ V_{1}^{1}(\Omega) & \text { if }|k| \geq 1\end{cases}
$$


provided with the norms and seminorms

$$
\|v\|_{H_{(k)}^{1}(\Omega)}=\left(\|v\|_{H_{1}^{1}(\Omega)}^{2}+|k|^{2}\|v\|_{L_{-1}^{2}(\Omega)}^{2}\right)^{\frac{1}{2}}, \quad|v|_{H_{(k)}^{1}(\Omega)}=\left(|v|_{H_{1}^{1}(\Omega)}^{2}+|k|^{2}\|v\|_{L_{-1}^{2}(\Omega)}^{2}\right)^{\frac{1}{2}} .
$$

We also define their subspaces

$$
H_{(k) \diamond}^{1}(\Omega)=H_{(k)}^{1}(\Omega) \cap H_{1 \diamond}^{1}(\Omega)
$$

Note that the equivalence of the norm $\|\cdot\|_{H_{(k)}^{1}(\Omega)}$ and seminorm $|\cdot|_{H_{(k)}^{1}(\Omega)}$ on $H_{(k) \diamond}^{1}(\Omega)$, which is obvious for $k \neq 0$, also holds for $k=0$ (see [10], Prop. II.4.1).

The interest of these $k$-dependent spaces is that, as proven in ([10], Thm. II.3.1) the mapping: $\breve{\mu} \mapsto\left(\mu^{k}\right)_{k \in \mathbb{Z}}$ is an isomorphism from $H^{1}(\breve{\Omega})$ onto $\prod_{k \in \mathbb{Z}} H_{(k)}^{1}(\Omega)$ and also from $H_{0}^{1}(\breve{\Omega})$ onto $\prod_{k \in \mathbb{Z}} H_{(k) \diamond}^{1}(\Omega)$. In the vectorial case, we recall from ([10], Thm. II.3.6) that the mapping: $\breve{\boldsymbol{v}} \mapsto\left(v_{r}^{k}, v_{\theta}^{k}, v_{z}^{k}\right)_{k \in \mathbb{Z}}$ is an isomorphism from $H^{1}(\breve{\Omega})^{3}$ onto $\prod_{k \in \mathbb{Z}} \boldsymbol{H}_{(k)}^{1}(\Omega)$, where the spaces $\boldsymbol{H}_{(k)}^{1}(\Omega)$ are defined by

$$
\boldsymbol{H}_{(k)}^{1}(\Omega)= \begin{cases}V_{1}^{1}(\Omega) \times V_{1}^{1}(\Omega) \times H_{1}^{1}(\Omega) & \text { if } k=0, \\ \left\{\left(v_{r}, v_{\theta}, v_{z}\right) \in H_{1}^{1}(\Omega) \times H_{1}^{1}(\Omega) \times V_{1}^{1}(\Omega) ; v_{r}+i k v_{\theta} \in L_{-1}^{2}(\Omega)\right\} & \text { if }|k|=1, \\ V_{1}^{1}(\Omega) \times V_{1}^{1}(\Omega) \times V_{1}^{1}(\Omega) & \text { if }|k| \geq 2 .\end{cases}
$$

Finally, we are interested with the spaces involving the $\operatorname{curl}_{k}$ operator. So, we first define $H\left(\mathbf{c u r l}{ }_{k}, \Omega\right)$ as the space of vector fields $\boldsymbol{v}$ in $L_{1}^{2}(\Omega)^{3}$ such that $\operatorname{curl}_{k} \boldsymbol{v}$ also belongs to $L_{1}^{2}(\Omega)^{3}$. The density of $\mathscr{D}(\bar{\Omega})^{3}$ in each $H\left(\operatorname{curl}_{k}, \Omega\right)$ is an easy consequence of the analogous three-dimensional result on $\breve{\Omega}$ (see [15], Chap. I, Thm. 2.10). When combined this density result with the Stokes formula, valid for smooth enough vector fields $\boldsymbol{v}$ and $\varphi$ on $\bar{\Omega}$,

$$
\int_{\Omega} \boldsymbol{v} \cdot \operatorname{curl}_{-k} \overline{\boldsymbol{\varphi}} r \mathrm{~d} r \mathrm{~d} z-\int_{\Omega} \operatorname{curl}_{k} \boldsymbol{v} \cdot \overline{\boldsymbol{\varphi}} r \mathrm{~d} r \mathrm{~d} z=\int_{\Gamma} \gamma_{T} \boldsymbol{v} \cdot \overline{\boldsymbol{\varphi}} r(\tau) \mathrm{d} \tau
$$

(here $r(\tau)$ stands for the $r$-coordinate of the point with tangential abscissa $\tau$ ), we prove that the trace operator $\gamma_{T}$ introduced in (3.6) is continuous from $H\left(\operatorname{curl}_{k}, \Omega\right)$ into the dual space of traces on $\Gamma$ of functions in $\boldsymbol{H}_{(k)}^{1}(\Omega)$.

So we are now in a position to define for each $k \in \mathbb{Z}$ the space

$$
X_{(k)}(\Omega)=\left\{\boldsymbol{v} \in H\left(\operatorname{curl}_{k}, \Omega\right) ; \gamma_{T} \boldsymbol{v}=0 \text { on } \Gamma\right\}
$$

it is provided with the natural norm of $H\left(\operatorname{curl}_{k}, \Omega\right)$ :

$$
\|\boldsymbol{v}\|_{X_{(k)}(\Omega)}=\left(\|\boldsymbol{v}\|_{L_{1}^{2}(\Omega)^{3}}^{2}+\left\|\operatorname{curl}_{k} \boldsymbol{v}\right\|_{L_{1}^{2}(\Omega)^{3}}^{2}\right)^{\frac{1}{2}}
$$

Next, we observe from formula (3.14) that the mapping: $\boldsymbol{v} \mapsto \operatorname{curl}_{k} \boldsymbol{v}$ is continuous from $L_{1}^{2}(\Omega)^{3}$ into the dual space of $X_{(k)}(\Omega)$, that we denote by $X_{(k)}(\Omega)^{\prime}$. This gives the idea for the definition of the space $Y_{(k)}(\Omega)$ :

$$
Y_{(k)}(\Omega)=\left\{\boldsymbol{v} \in L_{1}^{2}(\Omega)^{3} ; \operatorname{curl}_{k} \operatorname{curl}_{k} \boldsymbol{v} \in X_{(k)}(\Omega)^{\prime}\right\}
$$

The spaces $X_{(k)}(\Omega)$ and $Y_{(k)}(\Omega)$ are the basic ones for the variational formulation of problems (3.3) and (3.4). 


\subsection{Variational formulation and analysis of the first problem}

Since $\breve{\boldsymbol{f}}$ belongs to $L^{2}(\breve{\Omega})^{3}$, it is readily checked that each $\boldsymbol{f}^{k}, k \in \mathbb{Z}$, belongs to $L_{1}^{2}(\Omega)^{3}$. So, for each $k \in \mathbb{Z}$, we now propose the following variational formulation of problem (3.3):

$$
\begin{aligned}
& \text { Find } \boldsymbol{\omega}^{0 k} \text { in } X_{(k)}(\Omega) \text { and } \lambda^{0 k} \text { in } H_{(k) \diamond}^{1}(\Omega) \text { such that } \\
& \forall \boldsymbol{\varphi} \in X_{(k)}(\Omega), \quad a_{k}^{1}\left(\boldsymbol{\omega}^{0 k}, \boldsymbol{\varphi}\right)+b_{k}^{1}\left(\boldsymbol{\varphi}, \lambda^{0 k}\right)=\int_{\Omega} \boldsymbol{f}^{k} \cdot \operatorname{curl}_{-k} \overline{\boldsymbol{\varphi}} r \mathrm{~d} r \mathrm{~d} z, \\
& \forall \mu \in H_{(k) \diamond}^{1}(\Omega), \quad \overline{b_{k}^{1}}\left(\boldsymbol{\omega}^{0 k}, \mu\right)=0,
\end{aligned}
$$

where the sesquilinear forms $a_{k}^{1}(\cdot, \cdot)$ and $b_{k}^{1}(\cdot, \cdot)$ are defined by

$$
a_{k}^{1}(\boldsymbol{\vartheta}, \boldsymbol{\varphi})=\int_{\Omega} \operatorname{curl}_{k} \boldsymbol{\vartheta} \cdot \operatorname{curl}_{-k} \overline{\boldsymbol{\varphi}} r \mathrm{~d} r \mathrm{~d} z, \quad b_{k}^{1}(\boldsymbol{\varphi}, \mu)=\int_{\Omega} \operatorname{grad}_{k} \mu \cdot \overline{\boldsymbol{\varphi}} r \mathrm{~d} r \mathrm{~d} z
$$

These forms are obviously continuous on $X_{(k)}(\Omega) \times X_{(k)}(\Omega)$ and $X_{(k)}(\Omega) \times H_{(k)}^{1}(\Omega)$, respectively. Note also the formula

$$
\overline{b_{k}^{1}}(\varphi, \mu)=b_{-k}^{1}(\bar{\varphi}, \bar{\mu})
$$

Proposition 3.1. Any solution $\left(\boldsymbol{\omega}^{0 k}, \lambda^{0 k}\right)$ in $X_{(k)}(\Omega) \times H_{(k) \diamond}^{1}(\Omega)$ of problem (3.18) satisfies: $\lambda^{0 k}=0$ a.e. in $\Omega$ and is a solution of problem (3.3) in the distribution sense.

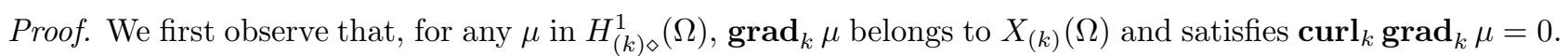
So, taking $\varphi$ equal to $\operatorname{grad}_{k} \lambda^{0 k}$ in $(3.18)$ yields

$$
\int_{\Omega}\left|\operatorname{grad}_{k} \lambda^{0 k}\right|^{2} r \mathrm{~d} r \mathrm{~d} z=0
$$

So, $\lambda^{0 k}$ is constant on $\Omega$ (which is connected) and, since it vanishes on $\Gamma$, it is equal to zero. As a consequence, taking $\varphi$ in (3.18) in $\mathscr{D}(\Omega)^{3}$ yields that the first line of (3.3) is satisfied in the distribution sense. Finally, letting $\mu$ in (3.18) run through $\mathscr{D}(\Omega)$ yields that the second line of (3.3) is satisfied in the distribution sense.

Note that the analysis of problem (3.18) can be derived from the three-dimensional results recalled in Section 2, however we have rather give a direct proof in view of its analogue for the discrete problem. As standard for saddle-point problems ([15], Chap. I, Thm. 4.1) proving the well-posedness of problem (3.18) relies on the ellipticity of $a_{k}^{1}(\cdot, \cdot)$ and on an inf-sup condition of Babuška and Brezzi type on the form $b_{k}^{1}(\cdot, \cdot)$. We begin with this condition.

Lemma 3.2. There exists a constant $\beta^{1}$ independent of $k$ such that, for each $k \in \mathbb{Z}$, the following inf-sup condition holds

$$
\forall \mu \in H_{(k) \diamond}^{1}(\Omega), \quad \sup _{\varphi \in X_{(k)}(\Omega)} \frac{b_{k}^{1}(\varphi, \mu)}{\|\varphi\|_{X_{(k)}(\Omega)}} \geq \beta^{1}|\mu|_{H_{(k)}^{1}(\Omega)} .
$$

Proof. Let $\mu$ be any function in $H_{(k) \diamond}^{1}(\Omega)$. As previously, the idea is to choose $\varphi$ equal to $\operatorname{grad}_{k} \mu$, so that

$$
b_{k}^{1}(\varphi, \mu)=\int_{\Omega}\left|\operatorname{grad}_{k} \mu\right|^{2} r \mathrm{~d} r \mathrm{~d} z=|\mu|_{H_{(k)}^{1}(\Omega)}^{2},
$$

and

This gives the desired inf-sup condition.

$$
\|\varphi\|_{X_{(k)}(\Omega)}=|\mu|_{H_{(k)}^{1}(\Omega)} .
$$


In order to check the ellipticity of the form $a_{k}^{1}(\cdot, \cdot)$, we introduce the kernel

$$
V_{(k)}^{1}=\left\{\boldsymbol{\varphi} \in X_{(k)}(\Omega) ; \forall \mu \in H_{(k) \diamond}^{1}(\Omega), \quad b_{k}^{1}(\boldsymbol{\varphi}, \mu)=0\right\} .
$$

By the same arguments as in the proof of Proposition 3.1, it is readily checked that

$$
V_{(k)}^{1}=\left\{\varphi \in X_{(k)}(\Omega) ; \operatorname{div}_{k} \varphi=0 \text { in } \Omega\right\} .
$$

Lemma 3.3. There exists a constant $\alpha^{1}$ independent of $k$ such that, for each $k \in \mathbb{Z}$, the following ellipticity property holds

$$
\forall \boldsymbol{\varphi} \in V_{(k)}^{1}, \quad a_{k}^{1}(\boldsymbol{\varphi}, \boldsymbol{\varphi}) \geq \alpha^{1}\|\boldsymbol{\varphi}\|_{X_{(k)}(\Omega)} .
$$

Proof. We have

$$
a_{k}^{1}(\boldsymbol{\varphi}, \boldsymbol{\varphi})=\left\|\operatorname{curl}_{k} \boldsymbol{\varphi}\right\|_{L_{1}^{2}(\Omega)^{3}}^{2} .
$$

In order to prove the equivalence of this last seminorm with the norm $\|\cdot\|_{X_{(k)}(\Omega)}$, we recall from ([5], Cor. 3.19) that there exists a constant $c_{1}$ such that, for all functions $\breve{\varphi}$ in $X(\breve{\Omega})$ with divergence in $L^{2}(\breve{\Omega})$,

$$
\|\breve{\varphi}\|_{L^{2}(\breve{\Omega})^{3}}^{2}+\|\operatorname{curl} \breve{\varphi}\|_{L^{2}(\breve{\Omega})^{3}}^{2}+\|\operatorname{div} \breve{\varphi}\|_{L^{2}(\breve{\Omega})}^{2} \leq c_{1}\left(\|\operatorname{curl} \breve{\varphi}\|_{L^{2}(\breve{\Omega})^{3}}^{2}+\|\operatorname{div} \breve{\varphi}\|_{L^{2}(\breve{\Omega})}^{2}\right) .
$$

Applying this inequality to the function $\breve{\varphi}$ such that its only non-zero Fourier coefficient is of order $k$ and belongs to $V_{(k)}^{1}$, yields

$$
\forall \boldsymbol{\varphi} \in V_{(k)}^{1}, \quad\|\boldsymbol{\varphi}\|_{L_{1}^{2}(\Omega)^{3}}^{2}+\left\|\operatorname{curl}_{k} \boldsymbol{\varphi}\right\|_{L_{1}^{2}(\Omega)^{3}}^{2} \leq c_{1}\left\|\operatorname{curl}_{k} \boldsymbol{\varphi}\right\|_{L_{1}^{2}(\Omega)^{3}}^{2},
$$

which implies the desired inequality.

Thanks to Lemmas 3.2 and 3.3, we now derive the next theorem from ([15], Chap. I, Thm. 4.1).

Theorem 3.4. For any data $f^{k}$ in $L_{1}^{2}(\Omega)^{3}$, problem (3.18) has a unique solution $\left(\boldsymbol{\omega}^{0 k}, \lambda^{0 k}\right)$ in $X_{(k)}(\Omega) \times$ $H_{(k) \diamond}^{1}(\Omega)$. Moreover, this solution satisfies: $\lambda^{0 k}=0$ a.e. in $\Omega$ and

$$
\left\|\boldsymbol{\omega}^{0 k}\right\|_{X_{(k)}(\Omega)} \leq c\left\|\boldsymbol{f}^{k}\right\|_{L_{1}^{2}(\Omega)^{3}} .
$$

Of course, since $\lambda^{0 k}$ is zero, the term $b_{k}^{1}\left(\boldsymbol{\varphi}, \lambda^{0 k}\right)$ could be suppressed in problem (3.18). However, it is important to keep it for the discrete problem that is built via the Galerkin method, in order to obtain a square linear system.

\subsection{Variational formulation and analysis of the second problem}

In analogy with Section 2 , for each $k \in \mathbb{Z}$, we propose the following variational formulation for problem (3.4):

$$
\begin{gathered}
\text { Find }\left(\boldsymbol{\omega}^{* k}, \lambda^{* k}\right) \text { in } Y_{(k)}(\Omega) \times H_{(k) \diamond}^{1}(\Omega) \text { and } \boldsymbol{\psi}^{k} \text { in } X_{(k)}(\Omega) \text { such that } \\
\forall(\boldsymbol{\vartheta}, \mu) \in Y_{(k)}(\Omega) \times H_{(k) \diamond}^{1}(\Omega), \quad a_{k}^{2}\left(\boldsymbol{\omega}^{* k}, \boldsymbol{\vartheta}\right)+b_{k}^{2}\left((\boldsymbol{\vartheta}, \mu) ; \boldsymbol{\psi}^{k}\right)=-a_{k}^{2}\left(\boldsymbol{\omega}^{0 k}, \boldsymbol{\vartheta}\right), \\
\forall \boldsymbol{\varphi} \in X_{(k)}(\Omega), \quad \overline{b_{k}^{2}}\left(\left(\boldsymbol{\omega}^{* k}, \lambda^{* k}\right) ; \boldsymbol{\varphi}\right)=0,
\end{gathered}
$$

where the sesquilinear forms $a_{k}^{2}(\cdot, \cdot)$ and $b_{k}^{2}(\cdot, \cdot)$ are now defined by

$$
a_{k}^{2}(\boldsymbol{\omega}, \boldsymbol{\vartheta})=\int_{\Omega} \boldsymbol{\omega} \cdot \overline{\boldsymbol{\vartheta}} r \mathrm{~d} r \mathrm{~d} z, \quad b_{k}^{2}((\boldsymbol{\vartheta}, \mu) ; \boldsymbol{\varphi})=-\left\langle\operatorname{curl}_{-k} \operatorname{curl}_{-k} \overline{\boldsymbol{\vartheta}}, \boldsymbol{\varphi}\right\rangle+\int_{\Omega} \boldsymbol{\varphi} \cdot \operatorname{grad}_{-k} \bar{\mu} r \mathrm{~d} r \mathrm{~d} z .
$$


There also, these forms are obviously continuous on $L_{1}^{2}(\Omega)^{3} \times L_{1}^{2}(\Omega)^{3}$ and on the product $\left(Y_{(k)}(\Omega) \times H_{(k)}^{1}(\Omega)\right) \times$ $X_{(k)}(\Omega)$. Moreover the form $a_{k}^{2}(\cdot, \cdot)$ is independent of $k$ and the form $\overline{b_{k}^{2}}(\cdot, \cdot)$ is simpler:

$$
\overline{b_{k}^{2}}((\boldsymbol{\vartheta}, \mu) ; \boldsymbol{\varphi})=-\left\langle\operatorname{curl}_{k} \operatorname{curl}_{k} \boldsymbol{\vartheta}, \overline{\boldsymbol{\varphi}}\right\rangle+\int_{\Omega} \operatorname{grad}_{k} \mu \cdot \overline{\boldsymbol{\varphi}} r \mathrm{~d} r \mathrm{~d} z .
$$

We now check the analogue of Proposition 3.1 for this problem.

Proposition 3.5. For any solution $\boldsymbol{\omega}^{0 k}$ of $(3.3)$ in $L_{1}^{2}(\Omega)^{3}$, any solution $\left(\boldsymbol{\omega}^{* k}, \lambda^{* k}, \boldsymbol{\psi}^{k}\right)$ in $Y_{(k)}(\Omega) \times H_{(k) \diamond}^{1}(\Omega) \times$ $X_{(k)}(\Omega)$ of problem (3.26) satisfies: $\lambda^{* k}=0$ a.e. in $\Omega$ and is a solution of problem (3.4) in the distribution sense.

Proof. We first take $\varphi$ equal to $\operatorname{grad}_{k} \lambda^{* k}$ in the second line of (3.26), which yields that

$$
\int_{\Omega}\left|\operatorname{grad}_{k} \lambda^{* k}\right|^{2} r \mathrm{~d} r \mathrm{~d} z=0
$$

or equivalently that $\lambda^{* k}$ is zero. Next, letting $\boldsymbol{\vartheta}$ run through $\mathscr{D}(\Omega)^{3}$ and taking $\mu=0$ in the first line of (3.26) gives the second line of (3.4), while letting $\varphi$ run through $\mathscr{D}(\Omega)^{3}$ in the second line of $(3.26)\left(\right.$ with $\left.\lambda^{* k}=0\right)$ yields the first equation of (3.4). Applying the $\operatorname{div}_{k}$ operator to the second line of (3.4) and using (3.3) yields that $\operatorname{div}_{k} \boldsymbol{\omega}^{* k}$ is zero, while the fact that $\operatorname{div}_{k} \boldsymbol{\psi}^{k}=0$ is obtained by taking $\boldsymbol{\vartheta}=\mathbf{0}$ and letting $\mu$ run through $\mathscr{D}(\Omega)$ in the first line of (3.26). Finally, the boundary condition $\gamma_{T}\left(\operatorname{curl}_{k} \psi^{k}\right)=0$ is derived from formula (3.14), by taking $\boldsymbol{\vartheta}$ equal to any infinitely differentiable function on $\bar{\Omega}$ and $\mu=0$ in the first line of (3.26).

In view of the analysis of problem (3.26), we prove the inf-sup condition on the form $b_{k}^{2}(\cdot, \cdot)$.

Lemma 3.6. There exists a constant $\beta^{2}$ independent of $k$ such that, for each $k \in \mathbb{Z}$, the following inf-sup condition holds

$$
\forall \boldsymbol{\varphi} \in X_{(k)}(\Omega), \quad \sup _{(\boldsymbol{\vartheta}, \mu) \in Y_{(k)}(\Omega) \times H_{(k) \diamond}^{1}(\Omega)} \frac{b_{k}^{2}((\boldsymbol{\vartheta}, \mu) ; \boldsymbol{\varphi})}{\|\boldsymbol{\vartheta}\|_{Y_{(k)}(\Omega)}+|\mu|_{H_{(k)}^{1}(\Omega)}} \geq \beta^{2}\|\boldsymbol{\varphi}\|_{X_{(k)}(\Omega)} .
$$

Proof. With any $\varphi$ in $X_{(k)}(\Omega)$, we associate the solution $\mu(\varphi)$ in $H_{(k) \diamond}^{1}(\Omega)$ of the problem

$$
\forall \mu \in H_{(k) \diamond}^{1}(\Omega), \quad \int_{\Omega} \operatorname{grad}_{k} \mu(\varphi) \cdot \operatorname{grad}_{-k} \bar{\mu} r \mathrm{~d} r \mathrm{~d} z=\int_{\Omega} \boldsymbol{\varphi} \cdot \operatorname{grad}_{-k} \bar{\mu} r \mathrm{~d} r \mathrm{~d} z .
$$

Such a problem is well-posed (see [10], Prop. II.4.1 and II.4.2). Next, we take $\boldsymbol{\vartheta}$ equal to $\boldsymbol{-} \boldsymbol{\varphi}$ (which belongs to $\left.Y_{(k)}(\Omega)\right)$ and $\mu$ equal to $\mu(\varphi)$, so that

$$
b_{k}^{2}((\boldsymbol{\vartheta}, \mu) ; \boldsymbol{\varphi})=\left\|\operatorname{curl}_{k} \boldsymbol{\varphi}\right\|_{L_{1}^{2}(\Omega)^{3}}^{2}+|\mu(\boldsymbol{\varphi})|_{H_{(k)}^{1}(\Omega)}^{2},
$$

and

Next, we use the triangle inequality

$$
\|\boldsymbol{\vartheta}\|_{Y_{(k)}(\Omega)}+|\mu|_{H_{(k)}^{1}(\Omega)} \leq\|\varphi\|_{X_{(k)}(\Omega)}+|\mu(\boldsymbol{\varphi})|_{H_{(k)}^{1}(\Omega)} .
$$

$$
\|\boldsymbol{\varphi}\|_{X_{(k)}(\Omega)} \leq\left\|\boldsymbol{\varphi}-\operatorname{grad}_{k} \mu(\boldsymbol{\varphi})\right\|_{L_{1}^{2}(\Omega)^{3}}+|\mu(\boldsymbol{\varphi})|_{H_{(k)}^{1}(\Omega)}+\left\|\operatorname{curl}_{k} \boldsymbol{\varphi}\right\|_{L_{1}^{2}(\Omega)^{3}},
$$

and, since the function $\boldsymbol{\varphi}-\operatorname{grad}_{k} \mu(\boldsymbol{\varphi})$ belongs to the space $V_{(k)}^{1}$ defined in (3.21), applying (3.24) yields

$$
\|\varphi\|_{X_{(k)}(\Omega)} \leq c_{1}\left\|\operatorname{curl}_{k} \boldsymbol{\varphi}\right\|_{L_{1}^{2}(\Omega)^{3}}+|\mu(\boldsymbol{\varphi})|_{H_{(k)}^{1}(\Omega)} .
$$

Combining (3.30) to (3.32) leads to the desired inf-sup condition. 
Next, we introduce the kernel

$$
V_{(k)}^{2}=\left\{(\boldsymbol{\vartheta}, \mu) \in Y_{(k)}(\Omega) \times H_{(k) \diamond}^{1}(\Omega) ; \forall \boldsymbol{\varphi} \in X_{(k)}(\Omega), \quad b_{k}^{2}((\boldsymbol{\vartheta}, \mu) ; \boldsymbol{\varphi})=0\right\} .
$$

We need a lemma to characterize this kernel.

Lemma 3.7. The space $V_{(k)}^{2}$ coincides with the space of pairs $(\boldsymbol{\vartheta}, \mu)$ in $Y_{(k)}(\Omega) \times H_{(k) \diamond}^{1}(\Omega)$ such that

$$
\operatorname{curl}_{k} \operatorname{curl}_{k} \vartheta=\mathbf{0} \text { and } \mu=0 \quad \text { a.e. in } \Omega \text {. }
$$

Proof. Let $(\boldsymbol{\vartheta}, \mu)$ be any pair in $V_{(k)}^{2}$. First, we take $\boldsymbol{\varphi}$ equal to $\operatorname{grad}_{k} \mu$ which belongs to $X_{(k)}(\Omega)$. This yields

$$
b_{k}^{2}((\boldsymbol{\vartheta}, \mu) ; \boldsymbol{\varphi})=\int_{\Omega}\left|\operatorname{grad}_{k} \mu\right|^{2} r \mathrm{~d} r \mathrm{~d} z=0
$$

so that $\mu$ is equal to zero. Next, we have

$$
\forall \varphi \in X_{(k)}(\Omega), \quad\left\langle\operatorname{curl}_{-k} \operatorname{curl}_{-k} \bar{\vartheta}, \boldsymbol{\varphi}\right\rangle=0,
$$

and since $\operatorname{curl}_{-k} \operatorname{curl}_{-k} \overline{\boldsymbol{\vartheta}}$ belongs to the dual space of $X_{(k)}(\Omega)$, it is zero.

We skip the proof of the ellipticity property of $a_{k}^{2}(\cdot, \cdot)$, which is now obvious.

Corollary 3.8. There exists a constant $\alpha^{2}$ independent of $k$ such that the following ellipticity property holds

$$
\forall(\boldsymbol{\vartheta}, \mu) \in V_{(k)}^{2}, \quad a_{k}^{2}(\boldsymbol{\vartheta}, \boldsymbol{\vartheta}) \geq \alpha^{2}\left(\|\boldsymbol{\vartheta}\|_{Y_{(k)}(\Omega)}^{2}+|\mu|_{H_{(k)}^{1}(\Omega)}^{2}\right) .
$$

The next theorem is now a consequence of ([15], Chap. I, Thm. 4.1) combined with Lemma 3.6 and Corollary 3.8.

Theorem 3.9. For any $\boldsymbol{\omega}^{0 k}$ in $L_{1}^{2}(\Omega)^{3}$, problem $(3.26)$ has a unique solution $\left(\boldsymbol{\omega}^{* k}, \lambda^{* k}, \boldsymbol{\psi}^{k}\right)$ in $Y_{(k)}(\Omega) \times$ $H_{(k) \diamond}^{1}(\Omega) \times X_{(k)}(\Omega)$. Moreover, this solution satisfies: $\lambda^{* k}=0$ a.e. in $\Omega$ and

$$
\left\|\boldsymbol{\omega}^{* k}\right\|_{L_{1}^{2}(\Omega)^{3}}+\left\|\boldsymbol{\psi}^{k}\right\|_{X_{(k)}(\Omega)} \leq c\left\|\boldsymbol{\omega}^{0 k}\right\|_{L_{1}^{2}(\Omega)^{3}}
$$

As previously, the term involving $\lambda^{* k}$ is kept in problem (3.26) only in order to obtain a discrete problem with as many unknowns as test functions. Moreover, on all pairs $(\boldsymbol{\vartheta}, \mu)$ such that $\boldsymbol{\vartheta}$ belongs to the smoother space $X_{(k)}(\Omega)$, the form $b_{k}^{2}(\cdot, \cdot)$ can equivalently be written

$$
b_{k}^{2}((\boldsymbol{\vartheta}, \mu) ; \boldsymbol{\varphi})=-a_{k}^{1}(\boldsymbol{\varphi}, \boldsymbol{\vartheta})+\overline{b_{k}^{1}}(\boldsymbol{\varphi}, \mu) .
$$

This simpler form is used in the discretization.

\subsection{Fourier truncation of the three-dimensional solution}

To conclude, we consider a fixed function $\breve{f}$ in $L^{2}(\breve{\Omega})^{3}$ with Fourier coefficients $\boldsymbol{f}^{k}, k \in \mathbb{Z}$. With each $\boldsymbol{f}^{k}$, we associate the unique solution $\left(\boldsymbol{\omega}^{0 k}, 0\right)$ of problem $(3.18)$ and the corresponding unique solution $\left(\boldsymbol{\omega}^{* k}, 0, \boldsymbol{\psi}^{k}\right)$ of problem (3.26). We set: $\boldsymbol{\omega}^{k}=\boldsymbol{\omega}^{0 k}+\boldsymbol{\omega}^{* k}$, and we define the three-dimensional functions $\boldsymbol{\omega}$ and $\boldsymbol{\psi}$ by

$$
\boldsymbol{\omega}(r, \theta, z)=\frac{1}{\sqrt{2 \pi}} \sum_{k \in \mathbb{Z}} \boldsymbol{\omega}^{k}(r, z) \mathrm{e}^{i k \theta}, \quad \boldsymbol{\psi}(r, \theta, z)=\frac{1}{\sqrt{2 \pi}} \sum_{k \in \mathbb{Z}} \boldsymbol{\psi}^{k}(r, z) \mathrm{e}^{i k \theta} .
$$

It is now readily checked that the corresponding pair $(\breve{\boldsymbol{\omega}}, \breve{\psi})$ is the only solution of problem $(2.4)$, so that the Stokes problem is fully equivalent to the system of problems $(3.18)$ and $(3.26), k \in \mathbb{Z}$. 
Remark. In the case of axisymmetric data $\breve{f}$, i.e. such that $f_{r}, f_{\theta}$ and $f_{z}$ are independent of $\theta$, all Fourier coefficients of $\breve{\omega}$ and $\breve{\psi}$ vanish but those of order zero. We refer to $[1,2]$ for a slightly different formulation of the problem in this case.

In the case of general data $\breve{f}$, the idea is to solve only a finite number of two-dimensional discrete problems. So, we fix an integer $K \geq 2$ and we introduce the pair $\left(\boldsymbol{\omega}_{K}, \boldsymbol{\psi}_{K}\right)$ which is obtained from $(\boldsymbol{\omega}, \boldsymbol{\psi})$ by Fourier truncation:

$$
\boldsymbol{\omega}_{K}(r, \theta, z)=\frac{1}{\sqrt{2 \pi}} \sum_{k=-K}^{K} \boldsymbol{\omega}^{k}(r, z) \mathrm{e}^{i k \theta}, \quad \boldsymbol{\psi}_{K}(r, \theta, z)=\frac{1}{\sqrt{2 \pi}} \sum_{k=-K}^{K} \boldsymbol{\psi}^{k}(r, z) \mathrm{e}^{i k \theta}
$$

We intend to evaluate the distance between $(\boldsymbol{\omega}, \boldsymbol{\psi})$ and $\left(\boldsymbol{\omega}_{K}, \boldsymbol{\psi}_{K}\right)$ in appropriate norms.

Estimating this distance relies on two arguments:

(i) Let $\pi_{K}$ be the standard Fourier truncation operator, defined from $L^{2}(-\pi, \pi)$ into itself by

$$
\varphi=\frac{1}{\sqrt{2 \pi}} \sum_{k \in \mathbb{Z}} \varphi^{k} \mathrm{e}^{i k \theta} \mapsto \pi_{K} \varphi=\frac{1}{\sqrt{2 \pi}} \sum_{k=-K}^{K} \varphi^{k} \mathrm{e}^{i k \theta}
$$

Then the following estimate is standard (see e.g. [11], Thm. 1.1): for any real numbers $s$ and $t$, $0 \leq t \leq s$, and for any function $\varphi$ in $H^{s}(-\pi, \pi)$,

$$
\left\|\varphi-\pi_{K} \varphi\right\|_{H^{t}(-\pi, \pi)} \leq c K^{t-s}\|\varphi\|_{H^{s}(-\pi, \pi)}
$$

(ii) The partial regularity of the solution of the Stokes problem with respect to $\theta$ only depends on the regularity of the data $\breve{f}$, but is independent of the regularity of the domain $\Omega$ (see [10], Prop. IX.1.8). We prove the next theorem by combining these arguments, thanks to the formula

$$
\omega-\omega_{K}=\omega-\pi_{K} \omega, \quad \operatorname{curl} \psi-\operatorname{curl} \psi_{K}=\operatorname{curl} \psi-\pi_{K}(\operatorname{curl} \psi) \text {. }
$$

Theorem 3.10. Assume that the data $\breve{\boldsymbol{f}}$ belong to $H^{\sigma}(\breve{\Omega})^{3}, \sigma \geq 0$. There exists a constant $c$ independent of $K$ such that the following estimate holds between the solution $(\breve{\boldsymbol{\omega}}, \breve{\boldsymbol{\psi}})$ of problem (2.4) and the pair $\left(\breve{\boldsymbol{\omega}}_{K}, \breve{\boldsymbol{\psi}}_{K}\right)$ defined in (3.39):

$$
\left\|\breve{\boldsymbol{\omega}}-\breve{\boldsymbol{\omega}}_{K}\right\|_{L^{2}(\breve{\Omega})^{3}}+K\left\|\operatorname{curl} \breve{\boldsymbol{\psi}}-\operatorname{curl} \breve{\boldsymbol{\psi}}_{K}\right\|_{L^{2}(\breve{\Omega})^{3}} \leq c K^{-\sigma-1}\|\breve{\boldsymbol{f}}\|_{H^{\sigma}(\breve{\Omega})^{3}}
$$

Remark. When the domain $\breve{\Omega}$ is convex, since both $\breve{\psi}$ and $\breve{\psi}_{K}$ are divergence-free and their tangential traces on $\partial \breve{\Omega}$ vanish, we deduce from ([5], Th. 2.17) that

$$
\left\|\breve{\psi}-\breve{\psi}_{K}\right\|_{H^{1}(\breve{\Omega})^{3}} \leq c\left\|\operatorname{curl} \breve{\psi}-\operatorname{curl} \breve{\psi}_{K}\right\|_{L^{2}(\breve{\Omega})^{3}}
$$

So, for convex domains $\breve{\Omega}$, we have also evaluated the distance between $\breve{\psi}$ and $\breve{\psi}_{K}$ in $H^{1}(\breve{\Omega})^{3}$.

\section{Spectral Discretization}

The Fourier coefficients of the data $\breve{f}$ cannot be computed explicitly in the general case. So we first explain how to compute them thanks to a quadrature formula. Next we present the polynomial spaces and the quadrature formulas that are needed for the discrete problems. We then describe the discretizations of problems (3.18) and (3.26) that are derived by the Galerkin method with numerical integration and we prove their 
well-posedness together with error estimates. In a final step, we construct a three-dimensional discrete solution from the previous problems.

In all that follows, we assume that $\breve{\Omega}$ is a cylinder. Equivalently, $\Omega$ is a rectangle and, without restriction, we take it equal to $\Omega=] 0,1[\times]-1,1\left[\right.$, with the corresponding definition for $\Gamma_{0}$ and $\Gamma$. More complex domains can be handled by combining the arguments in this paper with those of ([10], Chap. VIII).

\subsection{Fourier interpolation}

For the same integer $K$ as in Section 3.4, we introduce the $2 K+1$ equidistant nodes $\theta_{m}=\frac{2 m \pi}{2 K+1},-K \leq m \leq K$. Next, we assume the data $\breve{f}$ such that $f_{r}, f_{\theta}$ and $f_{z}$ are continuous with respect to $\theta$ and we set:

$$
\boldsymbol{f}_{\diamond}^{k}(r, z)=\frac{\sqrt{2 \pi}}{2 K+1} \sum_{m=-K}^{K} \boldsymbol{f}\left(t, \theta_{m}, z\right) \mathrm{e}^{-i k \theta_{m}}, \quad-K \leq k \leq K .
$$

Note that, clearly, these approximate Fourier coefficients depend on $K$, however we omit the $K$ in the index for simplicity. This leads to define the function

$$
\boldsymbol{f}_{K \diamond}(r, \theta, z)=\frac{1}{\sqrt{2 \pi}} \sum_{k=-K}^{K} \boldsymbol{f}_{\diamond}^{k}(r, z) \mathrm{e}^{i k \theta} .
$$

From the formula

$$
\boldsymbol{f}_{\diamond}^{k}=\sum_{\ell \in \mathbb{Z}} \boldsymbol{f}^{k+(2 K+1) \ell},
$$

it is readily checked that the function $\breve{\boldsymbol{f}}_{K \diamond}$ provides a good approximation of $\breve{\boldsymbol{f}}$, as stated in the next proposition.

Proposition 4.1. Assume the function $\breve{\boldsymbol{f}}$ in $H^{\sigma}(\breve{\Omega})^{3}, \sigma>\frac{1}{2}$. There exists a constant c independent of $K$ such that the following estimates hold:

$$
\left\|\breve{f}-\breve{f}_{K \diamond}\right\|_{L^{2}(\breve{\Omega})^{3}} \leq c K^{-\sigma}\|\breve{f}\|_{H^{\sigma}(\breve{\Omega})^{3}} \text { and }\left\|\breve{f}_{K \diamond}\right\|_{H^{\sigma}(\breve{\Omega})^{3}} \leq c\|\breve{f}\|_{H^{\sigma}(\breve{\Omega})^{3}} .
$$

Now, let $\left(\boldsymbol{\omega}_{\diamond}^{0 k}, \lambda_{\diamond}^{0 k}\right)$ be the solution of problem (3.18) with $\boldsymbol{f}^{k}$ replaced by $\boldsymbol{f}_{\diamond}^{k}$, and let $\left(\boldsymbol{\omega}_{\diamond}^{* k}, \lambda_{\diamond}^{* k}, \boldsymbol{\psi}_{\diamond}^{k}\right)$ be the solution of problem (3.26) with $\boldsymbol{\omega}^{0 k}$ replaced by $\boldsymbol{\omega}_{\diamond}^{0 k}$. It follows from the previous Proposition 4.1 combined with (3.25) and (3.36) that, if the function $\breve{f}$ belongs to $H^{\sigma}(\breve{\Omega})^{3}, \sigma>\frac{1}{2}$,

$$
\left(\sum_{k=-K}^{K}\left(\left\|\boldsymbol{\omega}^{0 k}-\boldsymbol{\omega}_{\diamond}^{0 k}\right\|_{X_{(k)}(\Omega)}^{2}+\left\|\boldsymbol{\omega}^{* k}-\boldsymbol{\omega}_{\diamond}^{* k}\right\|_{L_{1}^{2}(\Omega)^{3}}^{2}+\left\|\boldsymbol{\psi}^{k}-\boldsymbol{\psi}_{\diamond}^{k}\right\|_{X_{(k)}(\Omega)}^{2}\right)\right)^{\frac{1}{2}} \leq c K^{-\sigma}\|\breve{f}\|_{H^{\sigma}(\breve{\Omega})^{3}} .
$$

Moreover the uniqueness of the solutions of both problems (3.18) and (3.26), combined with (4.3), yields the formulas

$$
\boldsymbol{\omega}_{\diamond}^{0 k}=\sum_{\ell \in \mathbb{Z}} \boldsymbol{\omega}^{0, k+(2 K+1) \ell}, \quad \boldsymbol{\omega}_{\diamond}^{* k}=\sum_{\ell \in \mathbb{Z}} \boldsymbol{\omega}^{*, k+(2 K+1) \ell}, \quad \boldsymbol{\psi}_{\diamond}^{k}=\sum_{\ell \in \mathbb{Z}} \boldsymbol{\psi}^{k+(2 K+1) \ell},
$$

so that the regularity properties of the $\boldsymbol{\omega}_{\diamond}^{0 k}, \boldsymbol{\omega}_{\diamond}^{* k}$ and $\boldsymbol{\psi}_{\diamond}^{k}$ can be derived from those of $\breve{\boldsymbol{\omega}}^{0}, \breve{\boldsymbol{\omega}}^{*}$ and $\breve{\boldsymbol{\psi}}$ in an obvious way. 


\subsection{Polynomial spaces and quadrature formulas}

For each positive integer $n$, we define the space $\mathbb{P}_{n}(\Omega)$ as the space of polynomials on $\Omega$ with degree $\leq n$ with respect to $r$ and $z$, respectively. We introduce the three subspaces $\mathbb{P}_{n}^{*}(\Omega), \mathbb{P}_{n}^{\diamond}(\Omega)$ and $\mathbb{P}_{n}^{0}(\Omega)$ of polynomials in $\mathbb{P}_{n}(\Omega)$ which vanish on $\Gamma_{0}, \Gamma$ and $\partial \Omega$, respectively. We also use the space $\mathbb{P}_{n}(-1,1)$ of polynomials with degree $\leq n$ on $]-1,1$, its subspace $\mathbb{P}_{n}^{0}(-1,1)$ made of polynomials vanishing in \pm 1 , and their analogues on ]0, 1 [.

Let now $N$ be another fixed integer, $N \geqslant 2$. Our global discretization parameter is the pair $(K, N)$. We first define the space $\mathbb{M}_{N(k)}$ as the intersection of $\mathbb{P}_{N}(\Omega)$ with $H_{(k) \diamond}^{1}(\Omega)$. It is readily checked that

$$
\mathbb{M}_{N(k)}= \begin{cases}\mathbb{P}_{N}^{\diamond}(\Omega) & \text { if } k=0 \\ \mathbb{P}_{N}^{0}(\Omega) & \text { if }|k| \geq 1\end{cases}
$$

Similarly, we define $\mathbb{Y}_{N(k)}$ as the intersection of $\mathbb{P}_{N}(\Omega)^{3}$ with $H\left(\mathbf{c u r l}_{k}, \Omega\right)$ or, equivalently,

$$
\mathbb{Y}_{N(k)}= \begin{cases}\mathbb{P}_{N}(\Omega) \times \mathbb{P}_{N}^{*}(\Omega) \times \mathbb{P}_{N}(\Omega) & \text { if } k=0, \\ \left\{\left(v_{r N}, v_{\theta N}, v_{z N}\right) \in \mathbb{P}_{N}(\Omega) \times \mathbb{P}_{N}(\Omega) \times \mathbb{P}_{N}^{*}(\Omega) ; v_{\theta N}-i k v_{r N} \in \mathbb{P}_{N}^{*}(\Omega)\right\}, & \text { if }|k| \geq 1\end{cases}
$$

Finally, we introduce the space

$$
\mathbb{X}_{N(k)}=\left\{\boldsymbol{v}_{N} \in \mathbb{Y}_{N(k)} ; \gamma_{T} \boldsymbol{v}_{N}=0 \text { on } \Gamma\right\}
$$

Let $L_{n}$ stand for the Legendre polynomial of degree $n$. We recall that these polynomials are orthogonal to each other one in $L^{2}(-1,1)$ and satisfy $L_{n}(1)=1$. We now introduce the standard Gauss-Lobatto formula on ]-1,1[: With $\xi_{0}=-1$ and $\xi_{N}=1$, there exist a unique set of nodes $\xi_{j}, 1 \leq j \leq N-1$, and a unique set of weights $\rho_{j}, 0 \leq j \leq N$, such that the following property holds

$$
\forall \Phi \in \mathbb{P}_{2 N-1}(-1,1), \quad \int_{-1}^{1} \Phi(\xi) \mathrm{d} \xi=\sum_{j=0}^{N} \Phi\left(\xi_{j}\right) \rho_{j}
$$

The $\xi_{j}, 1 \leq j \leq N-1$, are the zeros of $L_{N}^{\prime}$ and the $\rho_{j}, 0 \leq j \leq N$, are positive. We finally recall the positivity property

$$
\forall \varphi_{N} \in \mathbb{P}_{N}(-1,1), \quad\left\|\varphi_{N}\right\|_{L^{2}(-1,1)}^{2} \leq \sum_{j=0}^{N} \varphi_{N}^{2}\left(\xi_{j}\right) \rho_{j} \leq 3\left\|\varphi_{N}\right\|_{L^{2}(-1,1)}^{2} .
$$

Similarly, the polynomials $M_{n}$ defined by

$$
M_{n}(\zeta)=\frac{L_{n}(\zeta)+L_{n+1}(\zeta)}{1+\zeta}, \quad n \geq 0
$$

are orthogonal to each other one on $]-1,1\left[\right.$ for the measure $(1+\zeta) \mathrm{d} \zeta$ and satisfy $M_{n}(1)=1$. The Gauss-Lobatto formula on ] $-1,1$ [ for the weighted measure $(1+\zeta) \mathrm{d} \zeta$ is defined as follows: With $\zeta_{0}=-1$ and $\zeta_{N}=1$, there exist a unique set of nodes $\zeta_{i}, 1 \leq i \leq N-1$, and a unique set of weights $\omega_{i}, 0 \leq i \leq N$, such that the following property holds

$$
\forall \Phi \in \mathbb{P}_{2 N-1}(-1,1), \quad \int_{-1}^{1} \Phi(\zeta)(1+\zeta) \mathrm{d} \zeta=\sum_{i=0}^{N} \Phi\left(\zeta_{i}\right) \omega_{i}
$$

The $\zeta_{i}, 1 \leq i \leq N-1$, are the zeros of $M_{N}^{\prime}$ and the $\omega_{i}, 0 \leq i \leq N$, are positive. We refer to ([10], Sect. VI.1.b) for a more detailed study of this formula and also for the positivity property

$$
\forall \varphi_{N} \in \mathbb{P}_{N}(-1,1), \quad\left\|\varphi_{N}(1+\zeta)^{\frac{1}{2}}\right\|_{L^{2}(-1,1)}^{2} \leq \sum_{i=0}^{N} \varphi_{N}^{2}\left(\zeta_{i}\right) \omega_{i} \leq 4\left\|\varphi_{N}(1+\zeta)^{\frac{1}{2}}\right\|_{L^{2}(-1,1)}^{2}
$$


Of course, we use formula (4.10) in the $z$-direction. In the $r$-direction, thanks to the change of variables $r=\frac{1+\zeta}{2}$, we use formula (4.13), so we set: $r_{i}=\frac{1+\zeta_{i}}{2}, 0 \leq i \leq N$. This leads to the definition of the discrete product, for any continuous functions $u$ and $v$ on $\bar{\Omega}$,

$$
((u, v))_{N}=\frac{1}{4} \sum_{i=0}^{N} \sum_{j=0}^{N} u\left(r_{i}, \xi_{j}\right) \bar{v}\left(r_{i}, \xi_{j}\right) \omega_{i} \rho_{j},
$$

and it can be extended to vector-valued functions in a trivial way. Let also $\mathcal{I}_{N}$ stands for the Lagrange interpolation operator at the nodes $\left(r_{i}, \xi_{j}\right), 0 \leq i, j \leq N$, with values in $\mathbb{P}_{N}(\Omega)$. We are now in a position to describe the discrete problems.

\subsection{Discretization of the first problem}

The discrete problem corresponding to problem (3.18) is obtained by the Galerkin method with numerical integration, it reads:

$$
\begin{gathered}
\text { Find } \boldsymbol{\omega}_{N}^{0 k} \text { in } \mathbb{X}_{N(k)} \text { and } \lambda_{N}^{0 k} \text { in } \mathbb{M}_{N(k)} \text { such that } \\
\forall \boldsymbol{\varphi}_{N} \in \mathbb{X}_{N(k)}, \quad a_{k N}^{1}\left(\boldsymbol{\omega}_{N}^{0 k}, \boldsymbol{\varphi}_{N}\right)+b_{k N}^{1}\left(\boldsymbol{\varphi}_{N}, \lambda_{N}^{0 k}\right)=\left(\left(\boldsymbol{f}_{\diamond}^{k}, \operatorname{curl}_{k} \boldsymbol{\varphi}_{N}\right)\right)_{N}, \\
\forall \mu_{N} \in \mathbb{M}_{N(k)}, \quad \overline{b_{k N}^{1}}\left(\boldsymbol{\omega}_{N}^{0 k}, \mu_{N}\right)=0,
\end{gathered}
$$

where the bilinear forms $a_{k N}^{1}(\cdot, \cdot)$ and $b_{k N}^{1}(\cdot, \cdot)$ are defined by

$$
a_{k N}^{1}\left(\boldsymbol{\vartheta}_{N}, \boldsymbol{\varphi}_{N}\right)=\left(\left(\operatorname{curl}_{k} \boldsymbol{\vartheta}_{N}, \operatorname{curl}_{k} \boldsymbol{\varphi}_{N}\right)\right)_{N}, \quad b_{k N}^{1}\left(\boldsymbol{\varphi}_{N}, \mu_{N}\right)=\left(\left(\operatorname{grad}_{k} \mu_{N}, \boldsymbol{\varphi}_{N}\right)\right)_{N} .
$$

There also, even if the function $\boldsymbol{f}_{\diamond}^{k}$ in the right-hand side depends on $K$, we omit the index $K$ and the $\diamond$ in $\boldsymbol{\omega}_{N}^{0 k}$ for simplicity.

Thanks to (4.11) and (4.14), the forms $a_{k N}^{1}(\cdot, \cdot)$ and $b_{k N}^{1}(\cdot, \cdot)$ satisfy the following continuity properties, with constants $c$ and $c^{\prime}$ independent of $k$ and $N$ :

$$
\begin{array}{ll}
\forall \boldsymbol{\vartheta}_{N} \in \mathbb{X}_{N(k)}, \forall \boldsymbol{\varphi}_{N} \in \mathbb{X}_{N(k)}, \quad a_{k N}^{1}\left(\boldsymbol{\vartheta}_{N}, \boldsymbol{\varphi}_{N}\right) \leq c\left\|\boldsymbol{\vartheta}_{N}\right\|_{X_{(k)}(\Omega)}\left\|\boldsymbol{\varphi}_{N}\right\|_{X_{(k)}(\Omega)}, \\
\forall \boldsymbol{\varphi}_{N} \in \mathbb{X}_{N(k)}, \forall \mu_{N} \in \mathbb{M}_{N(k)}, \quad b_{k N}^{1}\left(\boldsymbol{\varphi}_{N}, \mu_{N}\right) \leq c^{\prime}\left\|\boldsymbol{\varphi}_{N}\right\|_{X_{(k)}(\Omega)}\left|\mu_{N}\right|_{H_{(k)}^{1}(\Omega)} .
\end{array}
$$

The next lemma states a key property of our discrete spaces.

Lemma 4.2. For all $k \in \mathbb{Z}$, the $\operatorname{grad}_{k}$ operator maps $\mathbb{M}_{N(k)}$ into $\mathbb{X}_{N(k)}$.

Proof. For all $k$ and any $\mu_{N}$ in $\mathbb{M}_{N(k)}$, the function $\boldsymbol{v}_{N}=\operatorname{grad}_{k} \mu_{N}$ belongs to $\mathbb{P}_{N}(\Omega)^{3}$, so it remains to check that it satisfies the nullity conditions on $\Gamma_{0}$ and on $\Gamma$.

1) On $\Gamma_{0}$, for $k=0$, the angular component $v_{\theta N}$ is equal to zero, hence belongs to $\mathbb{P}_{N}^{*}(\Omega)$. For $k \neq 0$, we have

$$
v_{\theta N}-i k v_{r N}=i k r^{-1} \mu_{N}-i k \partial_{r} \mu_{N}=-i k r \partial_{r}\left(r^{-1} \mu_{N}\right) .
$$

Since $\mu_{N}$ belongs to $\mathbb{P}_{N}^{0}(\Omega)$, hence vanishes on $\Gamma_{0}$, the quantity $r^{-1} \mu_{N}$ belongs to $\mathbb{P}_{N}(\Omega)$. Thus, the polynomial $v_{\theta N}-i k v_{r N}$ vanishes on $\Gamma_{0}$, and the same property obviously holds for $v_{z N}=\partial_{z} \mu_{N}$.

2) On $\Gamma$, the quantities $\left(\gamma_{T} \boldsymbol{v}\right)_{r}=i k r^{-1} \mu_{N} n_{z}$ and $\left(\gamma_{T} \boldsymbol{v}\right)_{z}=-i k r^{-1} \mu_{N} n_{r}$ also vanish since $\mu_{N}$ has a zero trace on $\Gamma$. Moreover, we have

$$
\left(\gamma_{T} \boldsymbol{v}\right)_{\theta}=\partial_{z} \mu_{N} n_{r}-\partial_{r} \mu_{N} n_{z} .
$$

According to the edges of $\Gamma$, either $n_{r}$ is equal to $1, n_{z}$ is equal to zero and $\partial_{z}$ represents the tangential derivative or $n_{r}$ is equal to zero, $n_{z}$ is equal to \pm 1 and $\partial_{r}$ represents the tangential derivative. So, in all cases, $\left(\gamma_{T} \boldsymbol{v}\right)_{\theta}$ vanishes. 
Thanks to the Definition (4.9) of $\mathbb{X}_{N(k)}$, this concludes the proof.

We can now prove the inf-sup condition on the form $b_{k N}^{1}(\cdot, \cdot)$.

Lemma 4.3. There exists a constant $\tilde{\beta}^{1}$ independent of $k$ and $N$ such that, for each $k \in \mathbb{Z}$, the following inf-sup condition holds

$$
\forall \mu_{N} \in \mathbb{M}_{N(k)}, \quad \sup _{\varphi_{N} \in \mathbb{X}_{N(k)}} \frac{b_{k N}^{1}\left(\varphi_{N}, \mu_{N}\right)}{\left\|\varphi_{N}\right\|_{X_{(k)}}(\Omega)} \geq \tilde{\beta}^{1}\left|\mu_{N}\right|_{H_{(k)}^{1}(\Omega)} .
$$

Proof. Thanks to Lemma 4.2, the idea is to associate with any $\mu_{N}$ in $\mathbb{M}_{N(k)}$ the function $\varphi_{N}$ equal to $\operatorname{grad}_{k} \mu_{N}$. With this choice, we have

$$
b_{k N}^{1}\left(\varphi_{N}, \mu_{N}\right)=\left(\left(\operatorname{grad}_{k} \mu_{N}, \operatorname{grad}_{k} \mu_{N}\right)\right)_{N} .
$$

It follows from the positivity properties (4.11) and (4.14) that

$$
b_{k N}^{1}\left(\varphi_{N}, \mu_{N}\right) \geq\left|\mu_{N}\right|_{H_{(k)}^{1}(\Omega)}^{2},
$$

while

This concludes the proof.

$$
\left\|\varphi_{N}\right\|_{X_{(k)}(\Omega)}=\left|\mu_{N}\right|_{H_{(k)}^{1}(\Omega)}
$$

In order to prove the ellipticity of the form $a_{k N}^{1}(\cdot, \cdot)$, we introduce the kernel

$$
\mathbb{V}_{N(k)}^{1}=\left\{\boldsymbol{\varphi}_{N} \in \mathbb{X}_{N(k)} ; \forall \mu_{N} \in \mathbb{M}_{N(k)}, b_{k N}^{1}\left(\boldsymbol{\varphi}_{N}, \mu_{N}\right)=0\right\} .
$$

Lemma 4.4. There exists a constant $\tilde{\alpha}^{1}$ independent of $k$ and $N$ such that, for each $k \in \mathbb{Z}$, the following ellipticity property holds

$$
\forall \boldsymbol{\varphi}_{N} \in \mathbb{V}_{N(k)}^{1}, \quad a_{k N}^{1}\left(\varphi_{N}, \varphi_{N}\right) \geq \tilde{\alpha}^{1}\left\|\varphi_{N}\right\|_{X_{(k)}(\Omega)}^{2}
$$

To derive property (4.20), we only have to prove that

$$
\forall \boldsymbol{\varphi}_{N} \in \mathbb{V}_{N(k)}^{1}, \quad\left\|\boldsymbol{\varphi}_{N}\right\|_{L_{1}^{2}(\Omega)^{3}} \leq c\left\|\operatorname{curl}_{k} \boldsymbol{\varphi}_{N}\right\|_{L_{1}^{2}(\Omega)^{3}}
$$

Checking this inequality with a constant $c$ possibly depending on $N$ is easy. Indeed, let $\varphi_{N}$ be any polynomial in $\mathbb{V}_{N(k)}^{1}$ such that $\operatorname{curl}_{k} \boldsymbol{\varphi}_{N}$ is zero. The three-dimensional function such that its only non-zero coefficient is $\boldsymbol{\varphi}_{N}$ is also curl-free, so that it is the gradient of a function $\chi$. Then, it can be observed that the Fourier coefficient of order $k$ of $\chi$ belongs to $\mathbb{M}_{N(k)}$, hence is zero thanks to the definition of $\mathbb{V}_{N(k)}^{1}$. So, the right-hand side of (4.21) is a norm on $\mathbb{V}_{N(k)}^{1}$ and, from the equivalence of norms on finite-dimensional spaces, we derive (4.21) with a constant $c(N)$, possibly depending on $N$. Checking that the constant is independent of $N$ is rather technical, so we prefer to give the arguments in Appendix A.

Remark. As a by-product of the proof given in Appendix A, we note that $\mathbb{V}_{N(k)}^{1}$ is not contained in $V_{(k)}^{1}$, indeed all functions in $\mathbb{V}_{N(k)}^{1}$ are not exactly divergence-free. For instance, the function $\varphi_{N}$ given by

$$
\boldsymbol{\varphi}_{r N}(r, z)=0, \quad \boldsymbol{\varphi}_{\theta N}(r, z)=0, \quad \boldsymbol{\varphi}_{z N}=\mu_{N}(r) L_{N}(z)
$$

for any $\mu_{N}$ in $\mathbb{P}_{N}^{0}(0,1)$ belongs to $\mathbb{V}_{N(k)}^{1}$ and is not divergence-free.

Theorem 4.5. For any data $\boldsymbol{f}_{\diamond}^{k}$ continuous on $\bar{\Omega}$, problem (4.15) has a unique solution $\left(\boldsymbol{\omega}_{N}^{0 k}, \lambda_{N}^{0 k}\right)$ in $\mathbb{X}_{N(k)} \times$ $\mathbb{M}_{N(k)}$. Moreover, this solution satisfies: $\lambda_{N}^{0 k}=0$ in $\Omega$ and

$$
\left\|\boldsymbol{\omega}_{N}^{0 k}\right\|_{X_{(k)}(\Omega)} \leq c\left\|\mathcal{I}_{N} \boldsymbol{f}_{\diamond}^{k}\right\|_{L_{1}^{2}(\Omega)^{3}} .
$$


Proof. The existence and uniqueness of a solution are a direct consequence of Lemmas 4.3 and 4.4. Moreover, using once more Lemma 4.2, we take $\varphi_{N}$ equal to grad $\lambda_{N}^{0 k}$ in the first line of problem (4.15), which implies that $\lambda_{N}^{0 k}$ is zero. Finally, estimate (4.22) follows by taking $\boldsymbol{\varphi}_{N}$ equal to $\boldsymbol{\omega}_{N}^{0 k}$ in (4.15) and using Lemma 4.4 together with (4.11) and (4.14).

In order to prove the error estimate, we first check from Theorem 4.5 that

$$
\forall \boldsymbol{\varphi}_{N} \in \mathbb{X}_{N(k)}, \quad a_{k N}^{1}\left(\boldsymbol{\omega}_{N}^{0 k}, \boldsymbol{\varphi}_{N}\right)=\left(\left(\boldsymbol{f}_{\diamond}^{k}, \operatorname{curl}_{k} \boldsymbol{\varphi}_{N}\right)\right)_{N}
$$

Denoting by $\boldsymbol{\varphi}_{N}$ any approximation of $\boldsymbol{\omega}_{N}^{0 k}$ in $\mathbb{X}_{N-1(k)}$ (this space is defined by replacing the space $\mathbb{P}_{N}(\Omega)$ by $\mathbb{P}_{N-1}(\Omega)$ everywhere in the definition of $\left.\mathbb{X}_{N(k)}\right)$, we derive from (4.11) and (4.14) that

$$
\left\|\operatorname{curl}_{k}\left(\boldsymbol{\omega}_{N}^{0 k}-\boldsymbol{\varphi}_{N}\right)\right\|_{L_{1}^{2}(\Omega)^{3}}^{2} \leq a_{k N}^{1}\left(\boldsymbol{\omega}_{N}^{0 k}-\boldsymbol{\varphi}_{N}, \boldsymbol{\omega}_{N}^{0 k}-\boldsymbol{\varphi}_{N}\right)
$$

Combining (4.23) with the exactness property of the quadrature formula yields

$$
\left\|\operatorname{curl}_{k}\left(\boldsymbol{\omega}_{N}^{0 k}-\boldsymbol{\varphi}_{N}\right)\right\|_{L_{1}^{2}(\Omega)^{3}}^{2} \leq\left(\left(\boldsymbol{f}_{\diamond}^{k}, \operatorname{curl}_{k}\left(\boldsymbol{\omega}_{N}^{0 k}-\boldsymbol{\varphi}_{N}\right)\right)\right)_{N}-a_{k}^{1}\left(\boldsymbol{\varphi}_{N}, \boldsymbol{\omega}_{N}^{0 k}-\boldsymbol{\varphi}_{N}\right)
$$

Thus, it follows by adding (3.18) and using Theorem 3.4 that

$$
\begin{aligned}
\left\|\operatorname{curl}_{k}\left(\boldsymbol{\omega}_{N}^{0 k}-\boldsymbol{\varphi}_{N}\right)\right\|_{L_{1}^{2}(\Omega)^{3}}^{2} \leq a_{k}^{1}\left(\boldsymbol{\omega}_{\diamond}^{0 k}-\boldsymbol{\varphi}_{N}, \boldsymbol{\omega}_{N}^{0 k}-\boldsymbol{\varphi}_{N}\right) & \\
& -\int_{\Omega} \boldsymbol{f}_{\diamond}^{k} \cdot \operatorname{curl}_{-k}\left(\overline{\boldsymbol{\omega}}_{N}^{0 k}-\overline{\boldsymbol{\varphi}}_{N}\right) r \mathrm{~d} r \mathrm{~d} z+\left(\left(\boldsymbol{f}_{\diamond}^{k}, \operatorname{curl}_{k}\left(\boldsymbol{\omega}_{N}^{0 k}-\boldsymbol{\varphi}_{N}\right)\right)\right)_{N}
\end{aligned}
$$

By a triangle inequality, this leads to the estimate

$$
\begin{aligned}
\left\|\operatorname{curl}_{k}\left(\boldsymbol{\omega}_{\diamond}^{0 k}-\boldsymbol{\omega}_{N}^{0 k}\right)\right\|_{L_{1}^{2}(\Omega)^{3}} \leq & 2 \inf _{\boldsymbol{\varphi}_{N} \in \mathbb{X}_{N-1(k)}}\left\|\operatorname{curl}_{k}\left(\boldsymbol{\omega}_{\diamond}^{0 k}-\boldsymbol{\varphi}_{N}\right)\right\|_{L_{1}^{2}(\Omega)^{3}} \\
& +\sup _{\boldsymbol{\mu}_{N} \in \mathbb{P}_{N}(\Omega)^{3}} \frac{\int_{\Omega} \boldsymbol{f}_{\diamond}^{k} \cdot \overline{\boldsymbol{\mu}}_{N} r \mathrm{~d} r \mathrm{~d} z-\left(\left(\boldsymbol{f}_{\diamond}^{k}, \boldsymbol{\mu}_{N}\right)\right)_{N}}{\left\|\boldsymbol{\mu}_{N}\right\|_{L_{1}^{2}(\Omega)^{3}}} .
\end{aligned}
$$

The last term in (4.24) comes from numerical integration and is bounded in a standard way: for any function $\boldsymbol{f}$ continuous on $\bar{\Omega}$,

$$
\sup _{\boldsymbol{\mu}_{N} \in \mathbb{P}_{N}(\Omega)^{3}} \frac{\int_{\Omega} \boldsymbol{f} \cdot \overline{\boldsymbol{\mu}}_{N} r \mathrm{~d} r \mathrm{~d} z-\left(\left(\boldsymbol{f}, \boldsymbol{\mu}_{N}\right)\right)_{N}}{\left\|\boldsymbol{\mu}_{N}\right\|_{L_{1}^{2}(\Omega)^{3}}} \leq c\left(\left\|\boldsymbol{f}-\Pi_{N-1} \boldsymbol{f}\right\|_{L_{1}^{2}(\Omega)^{3}}+\left\|\boldsymbol{f}-\mathcal{I}_{N} \boldsymbol{f}\right\|_{L_{1}^{2}(\Omega)^{3}}\right),
$$

where $\Pi_{N-1}$ stands for the orthogonal projection operator from $L_{1}^{2}(\Omega)$ onto $\mathbb{P}_{N-1}(\Omega)$. Using ([10], Sect. V.2 and VI.3) leads to the following estimate: if the function $\boldsymbol{f}$ belongs to $H_{1}^{\sigma}(\Omega)^{3}, \sigma>\frac{3}{2}$,

$$
\sup _{\boldsymbol{\mu}_{N} \in \mathbb{P}_{N}(\Omega)^{3}} \frac{\int_{\Omega} \boldsymbol{f} \cdot \overline{\boldsymbol{\mu}}_{N} r \mathrm{~d} r \mathrm{~d} z-\left(\left(\boldsymbol{f}, \boldsymbol{\mu}_{N}\right)\right)_{N}}{\left\|\boldsymbol{\mu}_{N}\right\|_{L_{1}^{2}(\Omega)^{3}}} \leq c N^{-\sigma}\|\boldsymbol{f}\|_{H_{1}^{\sigma}(\Omega)^{3}}
$$

This is combined later with Proposition 4.1 in order to use the regularity properties of $\boldsymbol{f}^{k}$ instead of that of $\boldsymbol{f}_{\diamond}^{k}$.

The first term in the right-hand side of (4.24) represents the approximation error, and it is easy to bound it as a function of $\boldsymbol{\omega}_{\diamond}^{0 k}$. However, we have rather state a slightly improved result which is proved in Appendix B according to the arguments in [6], Section 4, and only involves the regularity of $\mathbf{c u r l}_{k} \boldsymbol{\omega}_{\diamond}^{0 k}$. 
Lemma 4.6. The following estimate holds for any function $\varphi$ in $X_{(k)}(\Omega)$ such that $\operatorname{curl}_{k} \varphi$ belongs to $H_{1}^{s}(\Omega)^{3}$, $s \geq 0$,

$$
\inf _{\varphi_{N} \in \mathbb{X}_{N(k)}}\left\|\operatorname{curl}_{k}\left(\varphi-\varphi_{N}\right)\right\|_{L_{1}^{2}(\Omega)^{3}} \leq c N^{-s}\left\|\operatorname{curl}_{k} \varphi\right\|_{H_{1}^{s}(\Omega)^{3}} .
$$

Inserting (4.25) and (4.26) into (4.24) leads to the first error estimate.

Theorem 4.7. Assume that the data $\boldsymbol{f}_{\diamond}^{k}$ belong to $H_{1}^{\sigma}(\Omega)^{3}, \sigma>\frac{3}{2}$, and that the vorticity $\boldsymbol{\omega}_{\diamond}^{0 k}$ of problem (3.18) is such that $\operatorname{curl}_{k} \boldsymbol{\omega}_{\diamond}^{0 k}$ belongs to $H_{1}^{s}(\Omega)^{3}, s \geq 0$. Then, the following error estimate holds

$$
\left\|\operatorname{curl}_{k}\left(\boldsymbol{\omega}_{\diamond}^{0 k}-\boldsymbol{\omega}_{N}^{0 k}\right)\right\|_{L_{1}^{2}(\Omega)^{3}} \leq c\left(N^{-s}\left\|\operatorname{curl}_{k} \boldsymbol{\omega}^{0 k}\right\|_{H_{1}^{s}(\Omega)^{3}}+N^{-\sigma}\left\|\boldsymbol{f}_{\diamond}^{k}\right\|_{H_{1}^{\sigma}(\Omega)^{3}}\right) .
$$

A better estimate for $\boldsymbol{\omega}_{\diamond}^{0 k}-\boldsymbol{\omega}_{N}^{0 k}$ in the $L_{1}^{2}(\Omega)^{3}$-norm can be derived by a duality argument.

Corollary 4.8. If the assumptions of Theorem 4.7 are satisfied, the following error estimate holds

$$
\left\|\boldsymbol{\omega}_{\diamond}^{0 k}-\boldsymbol{\omega}_{N}^{0 k}\right\|_{L_{1}^{2}(\Omega)^{3}} \leq c\left(N^{-1-s}\left\|\operatorname{curl}_{k} \boldsymbol{\omega}_{\diamond}^{0 k}\right\|_{H_{1}^{s}(\Omega)^{3}}+N^{-\sigma}\left\|\boldsymbol{f}_{\diamond}^{k}\right\|_{H_{1}^{\sigma}(\Omega)^{3}}\right) .
$$

Proof. We start from the formula

$$
\left\|\boldsymbol{\omega}_{\diamond}^{0 k}-\boldsymbol{\omega}_{N}^{0 k}\right\|_{L_{1}^{2}(\Omega)^{3}}=\sup _{\boldsymbol{g} \in L_{1}^{2}(\Omega)^{3}} \frac{\int_{\Omega}\left(\boldsymbol{\omega}_{\diamond}^{0 k}-\boldsymbol{\omega}_{N}^{0 k}\right) \cdot \overline{\boldsymbol{g}} r \mathrm{~d} r \mathrm{~d} z}{\|\boldsymbol{g}\|_{L_{1}^{2}(\Omega)^{3}}} .
$$

Next, for any $\boldsymbol{g}$ in $L_{1}^{2}(\Omega)^{3}$, we solve the problem

$$
\begin{cases}\operatorname{curl}_{k} \operatorname{curl}_{k} \boldsymbol{\varphi}=\boldsymbol{g} & \text { in } \Omega, \\ \operatorname{div}_{k} \boldsymbol{\varphi}=0 & \text { in } \Omega, \\ \gamma_{T} \boldsymbol{\varphi}=\mathbf{0} & \text { on } \Gamma .\end{cases}
$$

Its well-posedness follows from Theorem 3.4. Moreover, by noting that the $\operatorname{curl}_{k}$ of $\operatorname{curl}_{k} \varphi$ belongs to $L_{1}^{2}(\Omega)^{3}$, that its $\operatorname{div}_{k}$ is zero and that its normal trace vanishes on $\Gamma$ (which can easily be derived from the condition $\gamma_{T} \boldsymbol{\varphi}=\mathbf{0}$ ), we derive from the convexity of $\breve{\Omega}$ and the three-dimensional regularity results ([5], Thm. 2.17) that $\operatorname{curl}_{k} \varphi$ belongs to $H_{1}^{1}(\Omega)^{3}$ and satisfies

$$
\left\|\operatorname{curl}_{k} \boldsymbol{\varphi}\right\|_{H_{1}^{1}(\Omega)^{3}} \leq c\|\boldsymbol{g}\|_{L_{1}^{2}(\Omega)^{3}} .
$$

Moreover, we have

$$
\int_{\Omega}\left(\boldsymbol{\omega}_{\diamond}^{0 k}-\boldsymbol{\omega}_{N}^{0 k}\right) \cdot \overline{\boldsymbol{g}} r \mathrm{~d} r \mathrm{~d} z=a_{k}^{1}\left(\boldsymbol{\omega}_{\diamond}^{0 k}-\boldsymbol{\omega}_{N}^{0 k}, \boldsymbol{\varphi}\right) .
$$

Then, we derive from (4.23) that, for any $\boldsymbol{\varphi}_{N}$ in $\mathbb{X}_{N-1(k)}$,

$$
\int_{\Omega}\left(\boldsymbol{\omega}_{\diamond}^{0 k}-\boldsymbol{\omega}_{N}^{0 k}\right) \cdot \overline{\boldsymbol{g}} r \mathrm{~d} r \mathrm{~d} z=a_{k}^{1}\left(\boldsymbol{\omega}_{\diamond}^{0 k}-\boldsymbol{\omega}_{N}^{0 k}, \boldsymbol{\varphi}-\boldsymbol{\varphi}_{N}\right)+\int_{\Omega} \boldsymbol{f}_{\diamond}^{k} \cdot \operatorname{curl}_{-k} \overline{\boldsymbol{\varphi}}_{N} r \mathrm{~d} r \mathrm{~d} z-\left(\left(\boldsymbol{f}_{\diamond}^{k}, \operatorname{curl}_{k} \boldsymbol{\varphi}_{N}\right)\right)_{N}
$$

Finally, combining (4.25), Lemma 4.6 and (4.30) with the estimate of Theorem 4.7 leads to the desired result. 


\subsection{Discretization of the second problem}

The discrete problem corresponding to problem (3.26) now reads:

$$
\begin{aligned}
& \text { Find }\left(\boldsymbol{\omega}_{N}^{* k}, \lambda_{N}^{* k}\right) \text { in } \mathbb{Y}_{N(k)} \times \mathbb{M}_{N(k)} \text { and } \boldsymbol{\psi}_{N}^{k} \text { in } \mathbb{X}_{N(k)} \text { such that } \\
& \forall\left(\boldsymbol{\vartheta}_{N}, \mu_{N}\right) \in \mathbb{Y}_{N(k)} \times \mathbb{M}_{N(k)}, \\
& a_{k N}^{2}\left(\boldsymbol{\omega}_{N}^{* k}, \boldsymbol{\vartheta}_{N}\right)+b_{k N}^{2}\left(\left(\boldsymbol{\vartheta}_{N}, \mu_{N}\right) ; \boldsymbol{\psi}_{N}^{k}\right)=-a_{k N}^{2}\left(\boldsymbol{\omega}_{N}^{0 k}, \boldsymbol{\vartheta}_{N}\right), \\
& \forall \boldsymbol{\varphi}_{N} \in \mathbb{X}_{N(k)}, \quad \overline{b_{k N}^{2}}\left(\left(\boldsymbol{\omega}_{N}^{* k}, \lambda_{N}^{* k}\right) ; \boldsymbol{\varphi}_{N}\right)=0,
\end{aligned}
$$

where, as hinted in $(3.37)$, the bilinear forms $a_{k N}^{2}(\cdot, \cdot)$ and $b_{k N}^{2}(\cdot, \cdot)$ are now defined by

$$
a_{k N}^{2}\left(\boldsymbol{\omega}_{N}, \boldsymbol{\vartheta}_{N}\right)=\left(\left(\boldsymbol{\omega}_{N}, \boldsymbol{\vartheta}_{N}\right)\right)_{N}, \quad b_{k N}^{2}\left(\left(\boldsymbol{\vartheta}_{N}, \mu_{N}\right) ; \boldsymbol{\varphi}_{N}\right)=-a_{k N}^{1}\left(\boldsymbol{\varphi}_{N}, \boldsymbol{\vartheta}_{N}\right)+\overline{b_{k N}^{1}}\left(\boldsymbol{\varphi}_{N}, \mu_{N}\right) .
$$

Proving that this problem is well-posed there also relies on the inf-sup condition on $b_{k N}^{2}(\cdot ; \cdot)$ and on the ellipticity of $a_{k N}^{2}(\cdot, \cdot)$. We prove the first property by very similar arguments as in the continuous case.

Lemma 4.9. There exists a constant $\tilde{\beta}^{2}$ independent of $k$ and $N$ such that, for each $k \in \mathbb{Z}$, the following inf-sup condition holds

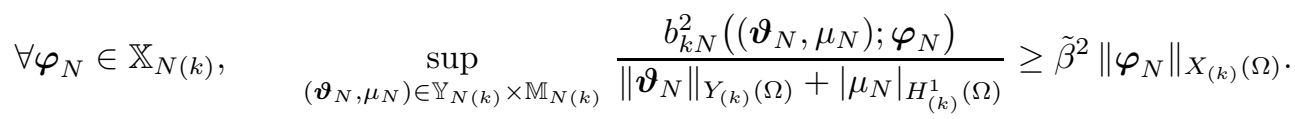

Proof. With any $\boldsymbol{\varphi}_{N}$ in $\mathbb{X}_{N(k)}$, we associate the solution $\mu_{N}\left(\boldsymbol{\varphi}_{N}\right)$ in $\mathbb{M}_{N(k)}$ of the problem

$$
\forall \lambda_{N} \in \mathbb{M}_{N(k)}, \quad \int_{\Omega} \operatorname{grad}_{k} \mu_{N}\left(\varphi_{N}\right) \cdot \operatorname{grad}_{-k} \bar{\lambda}_{N} r \mathrm{~d} r \mathrm{~d} z=\int_{\Omega} \boldsymbol{\varphi}_{N} \cdot \operatorname{grad}_{-k} \bar{\lambda}_{N} r \mathrm{~d} r \mathrm{~d} z .
$$

So, taking $\boldsymbol{\vartheta}_{N}$ equal to $-\boldsymbol{\varphi}_{N}$ and $\mu_{N}$ equal to $\mu_{N}\left(\boldsymbol{\varphi}_{N}\right)$ and using the positivity properties (4.11) and (4.14) yield

$$
b_{k N}^{2}\left(\left(\boldsymbol{\vartheta}_{N}, \mu_{N}\right) ; \boldsymbol{\varphi}_{N}\right) \geq\left\|\operatorname{curl}_{k} \boldsymbol{\varphi}_{N}\right\|_{L_{1}^{2}(\Omega)^{3}}^{2}+\left|\mu_{N}\left(\boldsymbol{\varphi}_{N}\right)\right|_{H_{(k)}^{1}(\Omega)}^{2} .
$$

On the other hand, we have

$$
\left\|\boldsymbol{\vartheta}_{N}\right\|_{Y_{(k)}(\Omega)}+\left|\mu_{N}\right|_{H_{(k)}^{1}(\Omega)} \leq\left\|\boldsymbol{\varphi}_{N}\right\|_{X_{(k)}(\Omega)}+\left|\mu_{N}\left(\boldsymbol{\varphi}_{N}\right)\right|_{H_{(k)}^{1}(\Omega)} .
$$

Owing to the triangle inequality

$$
\left\|\boldsymbol{\varphi}_{N}\right\|_{X_{(k)}(\Omega)} \leq\left\|\boldsymbol{\varphi}_{N}-\operatorname{grad}_{k} \mu_{N}\left(\boldsymbol{\varphi}_{N}\right)\right\|_{L_{1}^{2}(\Omega)^{3}}+\left|\mu_{N}\left(\boldsymbol{\varphi}_{N}\right)\right|_{H_{(k)}^{1}(\Omega)}+\left\|\operatorname{curl}_{k} \boldsymbol{\varphi}_{N}\right\|_{L_{1}^{2}(\Omega)^{3}}
$$

and using (4.21) (indeed, the function $\varphi_{N}-\operatorname{grad}_{k} \mu_{N}\left(\boldsymbol{\varphi}_{N}\right)$ belongs to the space $\mathbb{V}_{N(k)}^{1}$ defined in (4.19)), we derive

$$
\left\|\boldsymbol{\varphi}_{N}\right\|_{X_{(k)}(\Omega)} \leq\left\|\operatorname{curl}_{k} \boldsymbol{\varphi}_{N}\right\|_{L_{1}^{2}(\Omega)^{3}}+\left|\mu_{N}\left(\boldsymbol{\varphi}_{N}\right)\right|_{H_{(k)}^{1}(\Omega)},
$$

which, combined with (4.34) and (4.35), leads to the desired inf-sup condition.

The discrete kernel is now defined by

$$
\mathbb{V}_{N(k)}^{2}=\left\{\left(\boldsymbol{\vartheta}_{N}, \mu_{N}\right) \in \mathbb{Y}_{N(k)} \times \mathbb{M}_{N(k)} ; \forall \boldsymbol{\varphi}_{N} \in \mathbb{X}_{N(k)}, b_{k N}^{2}\left(\left(\boldsymbol{\vartheta}_{N}, \mu_{N}\right) ; \boldsymbol{\varphi}_{N}\right)=0\right\}
$$

The next property is an easy consequence of Lemma 4.2 . 
Lemma 4.10. All pairs $\left(\boldsymbol{\vartheta}_{N}, \mu_{N}\right)$ in $\mathbb{V}_{N(k)}^{2}$ are such that $\mu_{N}=0$ in $\Omega$.

Even if this result is not as precise as its analogue in the continuous case, see Lemma 3.7, using the equivalence of norms on finite-dimensional spaces leads to the following ellipticity property: There exists a constant $\alpha_{k N}^{2}$ depending on $k$ and $N$ such that the following inequality holds

$$
\forall\left(\boldsymbol{\vartheta}_{N}, \mu_{N}\right) \in \mathbb{V}_{N(k)}^{2}, \quad a_{k N}^{2}\left(\boldsymbol{\vartheta}_{N}, \boldsymbol{\vartheta}_{N}\right) \geq \alpha_{k N}^{2}\left\|\boldsymbol{\vartheta}_{N}\right\|_{Y_{(k)}(\Omega)}^{2}+\left|\mu_{N}\right|_{H_{(k)}^{1}(\Omega)}^{2} .
$$

This result is not optimal, but it is sufficient to derive the well-posedness result.

Theorem 4.11. For any data $\boldsymbol{\omega}_{N}^{0 k}$ in $\mathbb{P}_{N}(\Omega)^{3}$, problem (4.31) has a unique solution $\left(\boldsymbol{\omega}_{N}^{* k}, \lambda_{N}^{* k}, \boldsymbol{\psi}_{N}^{k}\right)$ in $\mathbb{Y}_{N(k)} \times$ $\mathbb{M}_{N(k)} \times \mathbb{X}_{N(k)}$. Moreover, this solution satisfies: $\lambda_{N}^{* k}=0$ in $\Omega$ and

$$
\left\|\boldsymbol{\omega}_{N}^{* k}\right\|_{L_{1}^{2}(\Omega)^{3}}+\left\|\boldsymbol{\psi}_{N}^{k}\right\|_{X_{(k)}(\Omega)} \leq c\left\|\boldsymbol{\omega}_{N}^{0 k}\right\|_{L_{1}^{2}(\Omega)^{3}} .
$$

Proof. The existence and uniqueness of the solution follow from Lemma 4.9 and (4.37). The fact that $\lambda_{N}^{* k}$ cancels can then be derived from Lemma 4.10, and the estimate on $\boldsymbol{\omega}_{N}^{* k}$ is obtained by taking $\boldsymbol{\vartheta}_{N}=\boldsymbol{\omega}_{N}^{* k}$ and $\mu_{N}=0$ in (4.31). The estimate on $\boldsymbol{\psi}_{N}^{k}$ follows from the inf-sup condition in Lemma 4.9.

In order to prove the error estimate, we introduce a fixed pair $\left(\boldsymbol{\vartheta}_{N}, 0\right)$ in $\mathbb{V}_{N(k)}^{2}$. Using the positivity properties (4.11) and (4.14) together with problem (4.31), we derive

$$
\begin{aligned}
\left\|\boldsymbol{\omega}_{N}^{* k}-\boldsymbol{\vartheta}_{N}\right\|_{L_{1}^{2}(\Omega)^{3}}^{2} & \leq a_{k N}^{2}\left(\boldsymbol{\omega}_{N}^{* k}-\boldsymbol{\vartheta}_{N}, \boldsymbol{\omega}_{N}^{* k}-\boldsymbol{\vartheta}_{N}\right) \\
& =-a_{k N}^{2}\left(\boldsymbol{\omega}_{N}^{0 k}, \boldsymbol{\omega}_{N}^{* k}-\boldsymbol{\vartheta}_{N}\right)-a_{k N}^{2}\left(\boldsymbol{\vartheta}_{N}, \boldsymbol{\omega}_{N}^{* k}-\boldsymbol{\vartheta}_{N}\right) .
\end{aligned}
$$

Thus, it follows from (3.26) that, for any $\varphi_{N}$ in $\mathbb{X}_{N-1(k)}$,

$$
\begin{aligned}
\left\|\boldsymbol{\omega}_{N}^{* k}-\boldsymbol{\vartheta}_{N}\right\|_{L_{1}^{2}(\Omega)^{3}}^{2} \leq a_{k}^{2}\left(\boldsymbol{\omega}_{\diamond}^{0 k}, \boldsymbol{\omega}_{N}^{* k}-\boldsymbol{\vartheta}_{N}\right)-a_{k N}^{2}\left(\boldsymbol{\omega}_{N}^{0 k}, \boldsymbol{\omega}_{N}^{* k}\right. & \left.-\boldsymbol{\vartheta}_{N}\right)+a_{k}^{2}\left(\boldsymbol{\omega}_{\diamond}^{* k}, \boldsymbol{\omega}_{N}^{* k}-\boldsymbol{\vartheta}_{N}\right) \\
& -a_{k N}^{2}\left(\boldsymbol{\vartheta}_{N}, \boldsymbol{\omega}_{N}^{* k}-\boldsymbol{\vartheta}_{N}\right)+b_{k}^{2}\left(\left(\boldsymbol{\omega}_{N}^{* k}-\boldsymbol{\vartheta}_{N}, 0\right), \boldsymbol{\psi}_{\diamond}^{k}-\boldsymbol{\varphi}_{N}\right) .
\end{aligned}
$$

As previously, we use the orthogonal projection operator $\Pi_{N-1}$ from $L_{1}^{2}(\Omega)$ onto $\mathbb{P}_{N-1}(\Omega)$ and we note for instance that

$$
\begin{aligned}
a_{k}^{2}\left(\boldsymbol{\omega}_{\diamond}^{0 k}, \boldsymbol{\omega}_{N}^{* k}-\boldsymbol{\vartheta}_{N}\right)-a_{k N}^{2}\left(\boldsymbol{\omega}_{N}^{0 k}, \boldsymbol{\omega}_{N}^{* k}-\boldsymbol{\vartheta}_{N}\right)=a_{k}^{2}\left(\boldsymbol{\omega}_{\diamond}^{0 k}-\Pi_{N-1} \boldsymbol{\omega}_{\diamond}^{0 k}, \boldsymbol{\omega}_{N}^{* k}-\boldsymbol{\vartheta}_{N}\right) & -a_{k N}^{2}\left(\boldsymbol{\omega}_{N}^{0 k}-\Pi_{N-1} \boldsymbol{\omega}_{\diamond}^{0 k}, \boldsymbol{\omega}_{N}^{* k}-\boldsymbol{\vartheta}_{N}\right) .
\end{aligned}
$$

So using a triangle inequality leads to

$$
\begin{aligned}
\left\|\boldsymbol{\omega}_{\diamond}^{* k}-\boldsymbol{\omega}_{N}^{* k}\right\|_{L_{1}^{2}(\Omega)^{3}} \leq & \left\|\boldsymbol{\omega}_{\diamond}^{0 k}-\boldsymbol{\omega}_{N}^{0 k}\right\|_{L_{1}^{2}(\Omega)^{3}}+\left\|\boldsymbol{\omega}_{\diamond}^{0 k}-\Pi_{N-1} \boldsymbol{\omega}_{\diamond}^{0 k}\right\|_{L_{1}^{2}(\Omega)^{3}} \\
& +\left\|\boldsymbol{\omega}_{\diamond}^{* k}-\boldsymbol{\vartheta}_{N}\right\|_{L_{1}^{2}(\Omega)^{3}}+\left\|\boldsymbol{\omega}_{\diamond}^{* k}-\Pi_{N-1} \boldsymbol{\omega}_{\diamond}^{* k}\right\|_{L_{1}^{2}(\Omega)^{3}}+\sup _{\boldsymbol{\zeta}_{N} \in \mathbb{Y}_{N(k)}} \frac{a_{k}^{1}\left(\boldsymbol{\psi}_{\diamond}^{k}-\boldsymbol{\varphi}_{N}, \boldsymbol{\zeta}_{N}\right)}{\left\|\boldsymbol{\zeta}_{N}\right\|_{L_{1}^{2}(\Omega)^{3}}} .
\end{aligned}
$$

Finally, we recall from ([15], Chap. II, formula (1.16)), combined with Lemmas 4.9 and 4.10 that

$$
\begin{aligned}
\inf _{\left(\boldsymbol{\vartheta}_{N}, 0\right) \in \mathbb{V}_{N(k)}^{2}}\left\|\boldsymbol{\omega}_{\diamond}^{* k}-\boldsymbol{\vartheta}_{N}\right\|_{L_{1}^{2}(\Omega)^{3}} & \leq \inf _{\left(\boldsymbol{\vartheta}_{N}, 0\right) \in \mathbb{V}_{N(k)}^{2}}\left\|\boldsymbol{\omega}_{\diamond}^{* k}-\boldsymbol{\vartheta}_{N}\right\|_{Y_{(k)}(\Omega)} \\
& \leq c \inf _{\boldsymbol{\vartheta}_{N} \in \mathbb{Y}_{N(k)}}\left\|\boldsymbol{\omega}_{\diamond}^{* k}-\boldsymbol{\vartheta}_{N}\right\|_{H\left(\operatorname{curl}_{k}, \Omega\right) .}
\end{aligned}
$$


Combining all this yields

$$
\begin{array}{r}
\left\|\boldsymbol{\omega}_{\diamond}^{* k}-\boldsymbol{\omega}_{N}^{* k}\right\|_{L_{1}^{2}(\Omega)^{3}} \leq\left\|\boldsymbol{\omega}_{\diamond}^{0 k}-\boldsymbol{\omega}_{N}^{0 k}\right\|_{L_{1}^{2}(\Omega)^{3}}+\left\|\boldsymbol{\omega}_{\diamond}^{0 k}-\Pi_{N-1} \boldsymbol{\omega}_{\diamond}^{0 k}\right\|_{L_{1}^{2}(\Omega)^{3}}+\inf _{\boldsymbol{\vartheta}_{N} \in \mathbb{Y}_{N(k)}}\left\|\boldsymbol{\omega}_{\diamond}^{* k}-\boldsymbol{\vartheta}_{N}\right\|_{H\left(\operatorname{curl}_{k}, \Omega\right)} \\
+\left\|\boldsymbol{\omega}_{\diamond}^{* k}-\Pi_{N-1} \boldsymbol{\omega}_{\diamond}^{* k}\right\|_{L_{1}^{2}(\Omega)^{3}}+\inf _{\boldsymbol{\varphi}_{N} \in \mathbb{X}_{N(k)} \operatorname{\zeta }_{N} \in \mathbb{Y}_{N(k)}} \frac{a_{k}^{1}\left(\boldsymbol{\psi}_{\diamond}^{k}-\boldsymbol{\varphi}_{N}, \boldsymbol{\zeta}_{N}\right)}{\left\|\boldsymbol{\zeta}_{N}\right\|_{L_{1}^{2}(\Omega)^{3}}} .
\end{array}
$$

The approximation result of functions in $H\left(\operatorname{curl}_{k}, \Omega\right)$ is stated in the next lemma which is proven in Appendix B.

Lemma 4.12. The following estimate holds for any function $\varphi$ in $Y_{(k)}(\Omega) \cap H_{1}^{s}(\Omega)^{3}, s>1$,

$$
\inf _{\boldsymbol{\varphi}_{N} \in \mathbb{Y}_{N(k)}}\left\|\boldsymbol{\varphi}-\boldsymbol{\varphi}_{N}\right\|_{H\left(\operatorname{curl}_{k}, \Omega\right)} \leq c N^{1-s}\|\boldsymbol{\varphi}\|_{H_{1}^{s}(\Omega)^{3}}
$$

The following estimate holds for any function $\varphi$ in $Y_{(k)}(\Omega) \cap H_{1}^{s}(\Omega)^{3}$, such that $\operatorname{curl}_{k} \varphi$ belongs to $H_{1}^{s}(\Omega)^{3}$, $s>3$,

$$
\inf _{\boldsymbol{\varphi}_{N} \in \mathbb{Y}_{N(k)}}\left\|\boldsymbol{\varphi}-\boldsymbol{\varphi}_{N}\right\|_{H\left(\operatorname{curl}_{k}, \Omega\right)} \leq c N^{-s}\left(\|\boldsymbol{\varphi}\|_{H_{1}^{s}(\Omega)^{3}}+\left\|\operatorname{curl}_{k} \boldsymbol{\varphi}\right\|_{H_{1}^{s}(\Omega)^{3}}\right) .
$$

The last term in (4.40) is evaluated in the next lemma, according to the idea in [8].

Lemma 4.13. Assume the function $\boldsymbol{\psi}_{\diamond}^{k}$ such that $\operatorname{curl}_{k} \boldsymbol{\psi}_{\diamond}^{k}$ belongs to $H_{1}^{t}(\Omega)^{3}, t \geq 1$. The following estimate holds

$$
\inf _{\boldsymbol{\varphi}_{N} \in \mathbb{X}_{N(k)}} \sup _{\boldsymbol{\zeta}_{N} \in \mathbb{Y}_{N(k)}} \frac{a_{k}^{1}\left(\boldsymbol{\psi}_{\diamond}^{k}-\boldsymbol{\varphi}_{N}, \boldsymbol{\zeta}_{N}\right)}{\left\|\boldsymbol{\zeta}_{N}\right\|_{L_{1}^{2}(\Omega)^{3}}} \leq c N^{\frac{3}{2}-t}\left\|\operatorname{curl}_{k} \boldsymbol{\psi}_{\diamond}^{k}\right\|_{H_{1}^{t}(\Omega)^{3}} .
$$

Proof. For any $\boldsymbol{\varphi}_{N}$ in $\mathbb{X}_{N(k)}$ such that $\gamma_{T}\left(\operatorname{curl}_{k} \boldsymbol{\varphi}_{N}\right)$ vanishes, we have thanks to an integration by parts

$$
a_{k}^{1}\left(\boldsymbol{\psi}_{\diamond}^{k}-\boldsymbol{\varphi}_{N}, \boldsymbol{\zeta}_{N}\right)=\int_{\Omega} \overline{\boldsymbol{\zeta}}_{N} \cdot \operatorname{curl}_{k} \operatorname{curl}_{k}\left(\boldsymbol{\psi}_{\diamond}^{k}-\boldsymbol{\varphi}_{N}\right) r \mathrm{~d} r \mathrm{~d} z
$$

whence

$$
\inf _{\boldsymbol{\varphi}_{N} \in \mathbb{X}_{N(k)}} \sup _{\boldsymbol{\zeta}_{N} \in \mathbb{Y}_{N(k)}} \frac{a_{k}^{1}\left(\boldsymbol{\psi}_{\diamond}^{k}-\boldsymbol{\varphi}_{N}, \boldsymbol{\zeta}_{N}\right)}{\left\|\boldsymbol{\zeta}_{N}\right\|_{L_{1}^{2}(\Omega)^{3}}} \leq c\left\|\operatorname{curl}_{k} \operatorname{curl}_{k}\left(\boldsymbol{\psi}_{\diamond}^{k}-\boldsymbol{\varphi}_{N}\right)\right\|_{L_{1}^{2}(\Omega)^{3}} .
$$

The desired result then follows from Appendix B, see Lemma B.5.

By combining the results of Lemmas 4.12 and 4.13 with (4.40) and also using Corollary 4.8 , we derive the first error estimate.

Theorem 4.14. Assume that the data $\boldsymbol{f}_{\diamond}^{k}$ belong to $H_{1}^{\sigma}(\Omega)^{3}, \sigma>\frac{3}{2}$, and that the vorticity $\boldsymbol{\omega}_{\diamond}^{0 k}$ of problem (3.18) belongs to $H_{1}^{s-1}(\Omega)^{3}, s \geq 1$, and is such that $\operatorname{curl}_{k} \boldsymbol{\omega}_{\diamond}^{0 k}$ belongs to $H_{1}^{s-1}(\Omega)^{3}$. Assume also that the solution $\left(\boldsymbol{\omega}_{\diamond}^{* k}, \psi_{\diamond}^{k}\right)$ of problem $(3.26)$ is such that $\boldsymbol{\omega}_{\diamond}^{* k}$ belongs to $H_{1}^{s}(\Omega)^{3}, s \geq 1$, and that $\operatorname{curl}_{k} \boldsymbol{\psi}_{\diamond}^{k}$ belongs to $H_{1}^{t}(\Omega)^{3}$, $t \geq 1$. Then, the following error estimate holds

$$
\begin{aligned}
\left\|\boldsymbol{\omega}_{\diamond}^{* k}-\boldsymbol{\omega}_{N}^{* k}\right\|_{L_{1}^{2}(\Omega)^{3}} \leq c\left(N ^ { 1 - s } \left(\left\|\boldsymbol{\omega}_{\diamond}^{0 k}\right\|_{H_{1}^{s-1}(\Omega)^{3}}\right.\right. & \left.+\left\|\operatorname{curl}_{k} \boldsymbol{\omega}_{\diamond}^{0 k}\right\|_{H_{1}^{s-1}(\Omega)^{3}}+\left\|\boldsymbol{\omega}_{\diamond}^{* k}\right\|_{H_{1}^{s}(\Omega)^{3}}\right) \\
& \left.+N^{\frac{3}{2}-t}\left\|\operatorname{curl}_{k} \boldsymbol{\psi}_{\diamond}^{k}\right\|_{H_{1}^{t}(\Omega)^{3}}+N^{-\sigma}\left\|\boldsymbol{f}_{\diamond}^{k}\right\|_{H_{1}^{\sigma}(\Omega)^{3}}\right) .
\end{aligned}
$$

This estimate and the following one are rather technical, but this is only due to the fact that we try to minimize the regularity properties of the solution which are required for them. Estimating the error between $\boldsymbol{\psi}_{\diamond}^{k}$ and $\boldsymbol{\psi}_{N}^{k}$ relies on the inf-sup condition (4.33). From now on, we set for simplicity:

$$
\boldsymbol{\omega}_{\diamond}^{k}=\boldsymbol{\omega}_{\diamond}^{0 k}+\boldsymbol{\omega}_{\diamond}^{* k}, \quad \boldsymbol{\omega}_{N}^{k}=\boldsymbol{\omega}_{N}^{0 k}+\boldsymbol{\omega}_{N}^{* k}
$$


Indeed, we derive from (4.33) that, for any $\varphi_{N}$ in $\mathbb{X}_{N-1(k)}$,

$$
\left\|\boldsymbol{\psi}_{N}^{k}-\boldsymbol{\varphi}_{N}\right\|_{X_{(k)}(\Omega)} \leq \tilde{\beta}_{2}^{-1} \sup _{\left(\boldsymbol{\vartheta}_{N}, \mu_{N}\right) \in \mathbb{Y}_{N(k)} \times \mathbb{M}_{N(k)}} \frac{b_{k N}^{2}\left(\left(\boldsymbol{\vartheta}_{N}, \mu_{N}\right) ; \boldsymbol{\psi}_{N}^{k}-\varphi_{N}\right)}{\left\|\boldsymbol{\vartheta}_{N}\right\|_{Y_{(k)}(\Omega)}+\left|\mu_{N}\right|_{H_{(k)}^{1}}(\Omega)}
$$

By using problems (3.26) and (4.31) together with the exactness properties of the quadrature formulas, we note that, for all $\left(\boldsymbol{\vartheta}_{N}, \mu_{N}\right)$ in $\mathbb{Y}_{N(k)} \times \mathbb{M}_{N(k)}$,

$b_{k N}^{2}\left(\left(\boldsymbol{\vartheta}_{N}, \mu_{N}\right) ; \boldsymbol{\psi}_{N}^{k}-\boldsymbol{\varphi}_{N}\right)=a_{k}^{2}\left(\boldsymbol{\omega}_{\diamond}^{k}-\Pi_{N-1} \boldsymbol{\omega}_{\diamond}^{k}, \boldsymbol{\vartheta}_{N}\right)+a_{k N}^{2}\left(\Pi_{N-1} \boldsymbol{\omega}_{\diamond}^{k}-\boldsymbol{\omega}_{N}^{k}, \boldsymbol{\vartheta}_{N}\right)+b_{k}^{2}\left(\left(\boldsymbol{\vartheta}_{N}, \mu_{N}\right) ; \boldsymbol{\psi}_{\diamond}^{k}-\boldsymbol{\varphi}_{N}\right)$.

Combining all this and using triangle inequalities leads to the estimate

$$
\left\|\boldsymbol{\psi}_{\diamond}^{k}-\boldsymbol{\psi}_{N}^{k}\right\|_{X_{(k)}(\Omega)} \leq c\left(\left\|\boldsymbol{\omega}_{\diamond}^{k}-\boldsymbol{\omega}_{N}^{k}\right\|_{L_{1}^{2}(\Omega)^{3}}+\left\|\boldsymbol{\omega}_{\diamond}^{k}-\Pi_{N-1} \boldsymbol{\omega}_{\diamond}^{k}\right\|_{L_{1}^{2}(\Omega)^{3}}+\inf _{\boldsymbol{\varphi}_{N} \in \mathbb{X}_{N-1(k)}}\left\|\boldsymbol{\psi}_{\diamond}-\boldsymbol{\varphi}_{N}\right\|_{X_{(k)}(\Omega)}\right) .
$$

So using a further triangle inequality relying on (4.45) for the terms involving $\boldsymbol{\omega}_{\diamond}$ together with Corollary 4.8 and Theorem 4.14, and also Lemma 4.6, we obtain the second error estimate.

Corollary 4.15. If the assumptions of Theorem 4.14 are satisfied, the following error estimate holds

$$
\begin{array}{r}
\left\|\boldsymbol{\psi}_{\diamond}^{k}-\boldsymbol{\psi}_{N}^{k}\right\|_{X_{(k)}(\Omega)} \leq c\left(N^{1-s}\left(\left\|\boldsymbol{\omega}_{\diamond}^{0 k}\right\|_{H_{1}^{s-1}(\Omega)^{3}}+\left\|\operatorname{curl}_{k} \boldsymbol{\omega}_{\diamond}^{0 k}\right\|_{H_{1}^{s-1}(\Omega)^{3}}+\left\|\boldsymbol{\omega}_{\diamond}^{* k}\right\|_{H_{1}^{s}(\Omega)^{3}}\right)\right. \\
\left.+N^{\frac{3}{2}-t}\left\|\operatorname{curl}_{k} \boldsymbol{\psi}_{\diamond}^{k}\right\|_{H_{1}^{t}(\Omega)^{3}}+N^{-\sigma}\left\|\boldsymbol{f}_{\diamond}^{k}\right\|_{H_{1}^{\sigma}(\Omega)^{3}}\right) .
\end{array}
$$

\subsection{The three-dimensional discrete solution}

The final idea is of course to associate with the solutions $\boldsymbol{\omega}_{N}^{0 k}$ of problem (4.15) and the solutions $\left(\boldsymbol{\omega}_{N}^{* k}, \boldsymbol{\psi}_{N}^{k}\right)$ of problem (4.31), $-K \leq k \leq K$, the three-dimensional pair $\left(\breve{\boldsymbol{\omega}}_{K N}, \breve{\boldsymbol{\psi}}_{K N}\right)$ defined by $\left(\boldsymbol{\omega}_{N}^{k}\right.$ is introduced in $(4.45))$

$$
\boldsymbol{\omega}_{K N}(r, \theta, z)=\frac{1}{\sqrt{2 \pi}} \sum_{k=-K}^{K} \boldsymbol{\omega}_{N}^{k}(r, z) \mathrm{e}^{i k \theta}, \quad \boldsymbol{\psi}_{K N}(r, \theta, z)=\frac{1}{\sqrt{2 \pi}} \sum_{k=-K}^{K} \boldsymbol{\psi}_{N}^{k}(r, z) \mathrm{e}^{i k \theta} .
$$

Indeed, we use the triangle inequality (with obvious definition of $\breve{\boldsymbol{\omega}}_{K \diamond}$ )

$$
\left\|\breve{\boldsymbol{\omega}}-\breve{\boldsymbol{\omega}}_{K N}\right\|_{L^{2}(\breve{\Omega})^{3}} \leq\left\|\breve{\boldsymbol{\omega}}-\breve{\boldsymbol{\omega}}_{K}\right\|_{L^{2}(\breve{\Omega})^{3}}+\left\|\breve{\boldsymbol{\omega}}_{K}-\breve{\boldsymbol{\omega}}_{K \diamond}\right\|_{L^{2}(\breve{\Omega})^{3}}+\left\|\breve{\boldsymbol{\omega}}_{K \diamond}-\breve{\boldsymbol{\omega}}_{K N}\right\|_{L^{2}(\breve{\Omega})^{3}}
$$

and a similar one for $\left\|\operatorname{curl}\left(\breve{\boldsymbol{\psi}}-\breve{\boldsymbol{\psi}}_{K N}\right)\right\|_{L^{2}(\breve{\Omega})^{3}}$. We also recall from Section 4.1 that the regularity properties of the $\breve{\boldsymbol{\omega}}^{0}, \breve{\boldsymbol{\omega}}^{*}, \breve{\boldsymbol{\psi}}$ and $\breve{\boldsymbol{f}}$ yield analogous ones for the $\boldsymbol{\omega}_{\diamond}^{0 k}, \boldsymbol{\omega}_{\diamond}^{* k}, \boldsymbol{\psi}_{\diamond}^{k}$ and $\boldsymbol{f}_{\diamond}^{k}$. So, the final estimate follows from Theorem 3.10, (4.5), Corollary 4.8, Theorem 4.14 and Corollary 4.15.

Theorem 4.16. Assume that

(i) the data $\breve{\boldsymbol{f}}$ belong to $H^{\sigma}(\breve{\Omega})^{3}, \sigma>\frac{3}{2}$;

(ii) the solution $\breve{\omega}^{0}$ of problem (2.9) belongs to $H^{s-1}(\breve{\Omega})^{3}, s \geq 1$, and is such that curl $\breve{\omega}^{0}$ belongs to $H^{s-1}(\breve{\Omega})^{3}$;

(iii) the solution $\left(\breve{\boldsymbol{\omega}}^{*}, \breve{\boldsymbol{\psi}}\right)$ of problem $(2.10)$ is such that $\breve{\boldsymbol{\omega}}^{*}$ belongs to $H_{1}^{s}(\Omega)^{3}, s \geq 1$, and that $\operatorname{curl} \breve{\boldsymbol{\psi}}$ belongs to $H^{t}(\breve{\Omega})^{3}, t \geq 0$. 
Then, the following error estimate holds between the solution $(\breve{\boldsymbol{\omega}}, \breve{\boldsymbol{\psi}})$ of problem $(2.4)$ and the pair $\left(\breve{\boldsymbol{\omega}}_{K N}, \breve{\boldsymbol{\psi}}_{K N}\right)$ defined in (4.48)

$$
\begin{aligned}
\left\|\breve{\boldsymbol{\omega}}-\breve{\boldsymbol{\omega}}_{K N}\right\|_{L^{2}(\breve{\Omega})^{3}}+\left\|\operatorname{curl}\left(\breve{\boldsymbol{\psi}}-\breve{\boldsymbol{\psi}}_{K N}\right)\right\|_{L^{2}(\Omega)^{3}} \leq & c\left(N^{1-s}\left(\left\|\breve{\boldsymbol{\omega}}^{0}\right\|_{H^{s-1}(\breve{\Omega})^{3}}+\left\|\operatorname{curl} \breve{\boldsymbol{\omega}}^{0}\right\|_{H^{s-1}(\breve{\Omega})^{3}}+\left\|\breve{\boldsymbol{\omega}}^{*}\right\|_{H^{s}(\breve{\Omega})^{3}}\right)\right. \\
& \left.+N^{\frac{3}{2}-t}\|\operatorname{curl} \breve{\boldsymbol{\psi}}\|_{H^{t}(\breve{\Omega})^{3}}+\left(K^{-\sigma}+N^{-\sigma}\right)\|\tilde{\boldsymbol{f}}\|_{H^{\sigma}(\breve{\Omega})^{3}}\right) . \quad(4.49)
\end{aligned}
$$

Remark. Assume that the data $\breve{f}$ belong to $H^{\sigma}(\breve{\Omega})^{3}, \sigma>\frac{3}{2}$. Thus combining (4.49) with the regularity properties stated in (2.11) to (2.13) leads to the estimate, for any positive $\varepsilon$,

$$
\left\|\breve{\boldsymbol{\omega}}-\breve{\boldsymbol{\omega}}_{K N}\right\|_{L^{2}(\breve{\Omega})^{3}}+\left\|\operatorname{curl}\left(\breve{\boldsymbol{\psi}}-\breve{\boldsymbol{\psi}}_{K N}\right)\right\|_{L^{2}(\Omega)^{3}} \leq c\left(K^{-\sigma}+N^{\varepsilon-1}\right)\|\breve{\boldsymbol{f}}\|_{H^{\sigma}(\breve{\Omega})^{3}} .
$$

So the convergence order is $\varepsilon-1$. However it seems likely that estimate (4.41) can be replaced by (4.42) in all the previous results, even for low values of $s$, which leads to a convergence order equal to $\varepsilon-2$. Moreover, the singular functions associated with problems (2.9) and (2.10) are explicitly known (see [10], Sect. II.4.c and IX.1.b) and, as usual, approximating these singular functions in a separate way leads to doubling the convergence order (see [10], Sect. 10.3).

Estimate (4.49) is not fully optimal in the general case but it proves the convergence of the method for all data in $H^{\sigma}(\breve{\Omega})^{3}, \sigma>\frac{3}{2}$, and, when $\sigma$ is large enough, $K$ can be chosen very small. So the previous discretization is not at all expensive in comparison with a three-dimensional computation and furthermore leads to an exactly divergence-free velocity $\breve{\boldsymbol{u}}_{K N}=\operatorname{curl} \breve{\boldsymbol{\psi}}_{K N}$.

\section{Appendix A}

The aim of this Appendix is to prove that inequality (4.21), i.e.

$$
\forall \varphi_{N} \in \mathbb{V}_{N(k)}^{1}, \quad\left\|\boldsymbol{\varphi}_{N}\right\|_{L_{1}^{2}(\Omega)^{3}} \leq c\left\|\operatorname{curl}_{k} \boldsymbol{\varphi}_{N}\right\|_{L_{1}^{2}(\Omega)^{3}},
$$

where the space $\mathbb{V}_{N(k)}^{1}$ is defined in (4.19), holds with a constant $c$ independent of $N$. We consider successively the cases $k=0$ and $k \neq 0$, since the arguments are rather different.

\section{Proof in the case $k=0$}

Let $\varphi_{N}$ be any polynomial in $\mathbb{V}_{N(0)}^{1}$. We handle separately the component $\varphi_{\theta N}$, next the components $\varphi_{r N}$ and $\varphi_{z N}$.

Indeed, since $\varphi_{\theta N}$ vanishes in $z=-1$, we have

$$
\varphi_{\theta N}^{2}(r, z)=\left(\int_{-1}^{z}\left(\partial_{z} \varphi_{\theta N}\right)(r, \zeta) \mathrm{d} \zeta\right)^{2} \leq 2 \int_{-1}^{1}\left(\partial_{z} \varphi_{\theta N}\right)^{2}(r, \zeta) \mathrm{d} \zeta
$$

Integrating this inequality on $\Omega$ with respect to the measure $r \mathrm{~d} r \mathrm{~d} z$ and noting that the radial component of $\operatorname{curl}_{0} \varphi_{N}$ coincides with $-\partial_{z} \varphi_{\theta N}$, we derive

$$
\left\|\varphi_{\theta N}\right\|_{L_{1}^{2}(\Omega)} \leq 2\left\|\left(\operatorname{curl}_{0} \varphi_{N}\right)_{r}\right\|_{L_{1}^{2}(\Omega)}
$$


Let us consider the expansions of $\varphi_{r N}$ and $\varphi_{z N}$ with respect to the polynomials $L_{n}$ and $M_{n}$. When taking into account the boundary conditions $\gamma_{T} \boldsymbol{\varphi}_{N}=\mathbf{0}$ on $\Gamma$, we have

$$
\begin{aligned}
& \varphi_{r N}(r, z)=\sum_{m=0}^{N} \sum_{n=1}^{N-1} \alpha_{m n} M_{m}(2 r-1)\left(L_{n+1}-L_{n-1}\right)(z), \\
& \varphi_{z N}(r, z)=\sum_{m=0}^{N-1} \sum_{n=0}^{N} \beta_{m n}\left(M_{m+1}-M_{m}\right)(2 r-1) L_{n}(z) .
\end{aligned}
$$

This yields

$$
\begin{aligned}
\left(\operatorname{div}_{0} \varphi_{N}\right)(r, z)=\sum_{m=0}^{N} \sum_{n=1}^{N-1} \alpha_{m n}\left(2 M_{m}^{\prime}+r^{-1} M_{m}\right)(2 r-1) & \left(L_{n+1}-L_{n-1}\right)(z) \\
& +\sum_{m=0}^{N-1} \sum_{n=0}^{N} \beta_{m n}\left(M_{m+1}-M_{m}\right)(2 r-1) L_{n}^{\prime}(z)
\end{aligned}
$$

It appears in this expansion that $\operatorname{div}_{0} \varphi_{N}$ vanishes in the two corners $(1,-1)$ and $(1,1)$ of $\Omega$ that are not on the axis $r=0$.

Next, using an integration by parts that relies on the exactness properties (4.10) and (4.13), we observe that the condition which defines $\mathbb{V}_{N(0)}^{1}$, see (4.19), can equivalently be written as

$$
\forall \mu_{N} \in \mathbb{P}_{N}^{\diamond}(\Omega), \quad\left(\left(\operatorname{div}_{0} \varphi_{N}, \mu_{N}\right)\right)_{N}=0
$$

This means that $\operatorname{div}_{0} \varphi_{N}$ vanishes in all nodes $\left(r_{i}, \xi_{j}\right), 0 \leq i, j \leq N$, that do not belong to $\Gamma$. So, there exist polynomials $\kappa_{N}^{-}$and $\kappa_{N}^{+}$in $\mathbb{P}_{N}(0,1)$ and $\lambda_{N}$ in $\mathbb{P}_{N}^{0}(-1,1)$ such that

$$
\left(\operatorname{div}_{0} \varphi_{N}\right)(r, z)=\kappa_{N}^{-}(r)(1-z) L_{N}^{\prime}(z)+\kappa_{N}^{+}(r)(1+z) L_{N}^{\prime}(z)+r M_{N}^{\prime}(2 r-1) \lambda_{N}(z)
$$

Moreover, since $\operatorname{div}_{0} \varphi_{N}$ vanishes in $(1, \pm 1)$, both $\kappa_{N}^{-}$and $\kappa_{N}^{+}$vanish in 1 .

The idea is now to compare (A.2) and (A.3). It appears that the second term of the right-hand side in (A.2) is orthogonal (for the discrete product) to $r M_{N}^{\prime}(2 r-1)$ and that the first term has degree $\leq N-1$ with respect to $r$, so that $\lambda_{N}$ is equal to zero. Similarly, the first term in (A.2) is orthogonal to $(1 \pm z) L_{N}^{\prime}(z)$ and the second has degree $\leq N-1$ with respect to $z$, whence the equality

$$
\kappa_{N}^{-}(r)=\kappa_{N}^{+}(r)=\frac{1}{2} \sum_{m=0}^{N-1} \beta_{m N}\left(M_{m+1}-M_{m}\right)(2 r-1) .
$$

It follows from the previous lines that the function $\left(\varphi_{r N}, 0, \varphi_{z N}\right)$ can be written as a sum $\varphi_{N}^{1}+\varphi_{N}^{2}$, where $\varphi_{N}^{1}$ is equal to $\left(0,0, \kappa_{N}(r) L_{N}(z)\right)$ for a polynomial $\kappa_{N}$ vanishing at $r=1$, while $\varphi_{N}^{2}=\left(\varphi_{r N}^{2}, 0, \varphi_{z N}^{2}\right)$ is exactly divergence-free and $\varphi_{z N}^{2}$ has degree $\leq N-1$ with respect to $z$. Clearly, we have

$$
\left\|\varphi_{z N}^{1}\right\|_{L_{1}^{2}(\Omega)}=\left\|\kappa_{N}\right\|_{L_{1}^{2}(0,1)}\left\|L_{N}\right\|_{L^{2}(-1,1)} \quad \text { and } \quad\left\|\partial_{r} \varphi_{z N}^{1}\right\|_{L_{1}^{2}(\Omega)}=\left\|\kappa_{N}^{\prime}\right\|_{L_{1}^{2}(0,1)}\left\|L_{N}\right\|_{L^{2}(-1,1)} .
$$

So applying the standard Poincaré-Friedrichs inequality to $\kappa_{N}$ (which vanishes at $r=1$ ) yields

$$
\left\|\varphi_{z N}^{1}\right\|_{L_{1}^{2}(\Omega)} \leq c\left\|\partial_{r} \varphi_{z N}^{1}\right\|_{L_{1}^{2}(\Omega)}=c\left\|\partial_{z} \varphi_{r N}^{1}-\partial_{r} \varphi_{z N}^{1}\right\|_{L_{1}^{2}(\Omega)} .
$$


Next, it follows from the previous lines that $\left(\varphi_{r N}^{2}, 0, \varphi_{z N}^{2}\right)$ belongs to the space $V_{(0)}^{1}$ introduced in $(3.21)$, so that applying Lemma 3.3 yields

$$
\left\|\varphi_{r N}^{2}\right\|_{L_{1}^{2}(\Omega)}+\left\|\varphi_{z N}^{2}\right\|_{L_{1}^{2}(\Omega)} \leq c\left\|\operatorname{curl}_{0} \varphi_{N}^{2}\right\|_{L_{1}^{2}(\Omega)^{3}}
$$

By noting that $\partial_{z} \varphi_{r N}^{1}-\partial_{r} \varphi_{z N}^{1}$ and $\partial_{z} \varphi_{r N}^{2}-\partial_{r} \varphi_{z N}^{2}$ (which has degree $\leq N-1$ with respect to $z$ ) are orthogonal in $L_{1}^{2}(\Omega)$, we thus derive

$$
\left\|\varphi_{r N}\right\|_{L_{1}^{2}(\Omega)}+\left\|\varphi_{z N}\right\|_{L_{1}^{2}(\Omega)} \leq c\left\|\operatorname{curl}_{0} \varphi_{N}\right\|_{L_{1}^{2}(\Omega)^{3}} .
$$

So the desired result for $k=0$ follows by combining (A.1) and (A.6).

\section{Proof in the case $k \neq 0$}

Let $\varphi_{N}=\left(\varphi_{r N}, \varphi_{\theta N}, \varphi_{z N}\right)$ be any polynomial in $\mathbb{V}_{N(k)}^{1}, k \neq 0$. Thanks to the definition (4.9) of the space $\mathbb{X}_{N(k)}$, it admits the expansion

$$
\begin{aligned}
& \varphi_{r N}(r, z)=\sum_{m=0}^{N} \sum_{n=1}^{N-1} \alpha_{m n} M_{m}(2 r-1)\left(L_{n+1}-L_{n-1}\right)(z), \\
& \varphi_{\theta N}(r, z)=\sum_{m=0}^{N-1} \sum_{n=1}^{N-1} \beta_{m n}\left(M_{m+1}-M_{m}\right)(2 r-1)\left(L_{n+1}-L_{n-1}\right)(z), \\
& \varphi_{z N}(r, z)=\sum_{m=0}^{N-2} \sum_{n=0}^{N} \gamma_{m n} r\left(M_{m+1}-M_{m}\right)(2 r-1) L_{n}(z),
\end{aligned}
$$

with the further condition

$$
\sum_{m=0}^{N-1} \beta_{m n}\left(M_{m+1}-M_{m}\right)(-1)-i k \sum_{m=0}^{N} \alpha_{m n} M_{m}(-1)=0 .
$$

This of course yields

$$
\begin{aligned}
\left(\operatorname{div}_{k} \varphi_{N}\right)(r, z)= & \sum_{m=0}^{N} \sum_{n=1}^{N-1} \alpha_{m n}\left(2 M_{m}^{\prime}+r^{-1} M_{m}\right)(2 r-1)\left(L_{n+1}-L_{n-1}\right)(z) \\
& +i k \sum_{m=0}^{N-1} \sum_{n=1}^{N-1} \beta_{m n} r^{-1}\left(M_{m+1}-M_{m}\right)(2 r-1)\left(L_{n+1}-L_{n-1}\right)(z) \\
& +\sum_{m=0}^{N-2} \sum_{n=0}^{N} \gamma_{m n} r\left(M_{m+1}-M_{m}\right)(2 r-1) L_{n}^{\prime}(z) .
\end{aligned}
$$

From this formula, $\operatorname{div}_{k} \varphi_{N}$ vanishes at the four corners of $\Omega$. On the other hand, the same arguments as previously yield that there exist four polynomials $\kappa_{N}^{-}$and $\kappa_{N}^{+}$in $\mathbb{P}_{N}^{0}(0,1), \lambda_{N}^{-}$and $\lambda_{N}^{+}$in $\mathbb{P}_{N}^{0}(-1,1)$ such that

$\left(\operatorname{div}_{k} \varphi_{N}\right)(r, z)=\kappa_{N}^{-}(r)(1-z) L_{N}^{\prime}(z)+\kappa_{N}^{+}(r)(1+z) L_{N}^{\prime}(z)+(1-r) M_{N}^{\prime}(r) \lambda_{N}^{-}(z)+r M_{N}^{\prime}(r) \lambda_{N}^{+}(z)$

There also the idea is to compare (A.7) and (A.8). As previously, we easily derive that

$$
\kappa_{N}^{-}(r)=\kappa_{N}^{+}(r)=\frac{1}{2} \sum_{m=0}^{N-2} \gamma_{m N} r\left(M_{m+1}-M_{m}\right)(2 r-1),
$$


and also that $\lambda_{N}^{-}$and $\lambda_{N}^{+}$are zero.

The end of the proof is nearly the same as in the case $k=0$. The function $\varphi_{N}$ can be written as a sum $\varphi_{N}^{1}+\varphi_{N}^{2}$, where $\varphi_{N}^{1}$ is equal to $\left(0,0, \kappa_{N}(r) L_{N}(z)\right)$ and the polynomial $\kappa_{N}$ vanishes at $r=0$ and $r=1$, while $\varphi_{N}^{2}$ is exactly divergence-free and its axial component $\varphi_{z N}^{2}$ has degree $\leq N-1$ with respect to $z$. The standard Poincaré-Friedrichs inequality applied to $\kappa_{N}$ yields

$$
\left\|\varphi_{z N}^{1}\right\|_{L_{1}^{2}(\Omega)} \leq c\left\|\partial_{r} \varphi_{z N}^{1}\right\|_{L_{1}^{2}(\Omega)}=c\left\|\partial_{z} \varphi_{r N}^{1}-\partial_{r} \varphi_{z N}^{1}\right\|_{L_{1}^{2}(\Omega)} .
$$

Next, it follows from Lemma 3.3 that

$$
\left\|\varphi_{N}^{2}\right\|_{L_{1}^{2}(\Omega)^{3}} \leq c\left\|\operatorname{curl}_{k} \varphi_{N}^{2}\right\|_{L_{1}^{2}(\Omega)^{3}} .
$$

We conclude by noting that $\operatorname{curl}_{k} \varphi_{N}^{1}$ and $\operatorname{curl}_{k} \boldsymbol{\varphi}_{N}^{2}$ are orthogonal in $L_{1}^{2}(\Omega)^{3}$.

\section{Appendix B}

The aim of this Appendix is to prove Lemmas 4.6 and 4.12, namely to establish the approximation properties of the space $\mathbb{X}_{N(k)}$ for functions in $X_{(k)}(\Omega)$ and also of the space $\mathbb{Y}_{N(k)}$ for functions in $Y_{(k)}(\Omega)$. We use two steps for this.

\section{Approximation of smooth functions}

In the $z$-direction, we consider the orthogonal projection operator $\pi_{N-1}^{z}$ from $L^{2}(-1,1)$ onto $\mathbb{P}_{N-1}(-1,1)$ and also the projection operator $\pi_{N}^{1 z}$ from $H^{1}(-1,1)$ onto $\mathbb{P}_{N}(-1,1)$ which preserves the values in \pm 1 and such that

$$
\forall \varphi \in H^{1}(-1,1), \quad\left(\pi_{N}^{1 z} \varphi\right)^{\prime}=\pi_{N-1}^{z} \varphi^{\prime} .
$$

In the $r$-direction, we use the same projection operators as $\pi_{N-1}^{z}$ and $\pi_{N}^{1 z}$, now translated onto ]0,1[, and we denote them by $\pi_{N-1}^{r}$ and $\pi_{N}^{1 r}$, respectively. We also introduce the analogue $\pi_{N-1}^{1 r}$ of $\pi_{N}^{1 r}$ with $N$ replaced by $N-1$ and the projection operator $\pi_{N}^{2 r}$ from $H^{2}(0,1)$ onto $\mathbb{P}_{N}(0,1)$ which preserves the values of the function and of its first derivative in 0 and 1 and such that

$$
\forall \varphi \in H^{2}(0,1), \quad\left(\pi_{N}^{2 r} \varphi\right)^{\prime}=\pi_{N-1}^{1 r} \varphi^{\prime} .
$$

Let now $\varphi$ be any smooth enough vector field on $\Omega$, with components $\varphi_{r}, \varphi_{\theta}$ and $\varphi_{z}$. The idea is to define the vector field $\Pi_{N}^{c} \varphi$ with components $\left(\Pi_{N}^{c} \varphi\right)_{r},\left(\Pi_{N}^{c} \varphi\right)_{\theta}$ and $\left(\Pi_{N}^{c} \varphi\right)_{z}$ by the formula

$$
\left(\Pi_{N}^{c} \varphi\right)_{r}=\pi_{N-1}^{1 r} \circ \pi_{N}^{1 z} \varphi_{r}, \quad\left(\Pi_{N}^{c} \varphi\right)_{\theta}=r^{-1} \pi_{N}^{2 r} \circ \pi_{N}^{1 z}\left(r \varphi_{\theta}\right), \quad\left(\Pi_{N}^{c} \varphi\right)_{z}=\pi_{N}^{2 r} \circ \pi_{N-1}^{z} \varphi_{z} .
$$

The idea for the choice of this operator, due to ([6], Sect. 4), in the Cartesian case, is that

1) for any smooth enough function $\varphi$, it preserves the nullity at $r=0$ of $\varphi_{\theta}$ for $k=0$, of $\varphi_{z}$ and $\varphi_{\theta}-i k \varphi_{r}$ for $|k| \geq 1$, so that it maps smooth functions in $H\left(\operatorname{curl}_{k}, \Omega\right)$ into $\mathbb{Y}_{N(k)}$;

2) for any function $\varphi$ such that $\gamma_{T} \varphi$ vanishes, so does $\gamma_{T}\left(\Pi_{N}^{c} \varphi\right)$, so that it maps smooth functions in $X_{(k)}(\Omega)$ into $\mathbb{X}_{N(k)}$;

3) the following property can be derived from (B.1) and (B.2)

$$
\begin{gathered}
\left(\operatorname{curl}_{k} \Pi_{N}^{c} \boldsymbol{\varphi}\right)_{r}=\tilde{\pi}_{N}^{2 r} \circ \pi_{N-1}^{z}\left(\operatorname{curl}_{k} \boldsymbol{\varphi}\right)_{r}, \quad\left(\operatorname{curl}_{k} \Pi_{N}^{c} \boldsymbol{\varphi}\right)_{\theta}=\pi_{N-1}^{1 r} \circ \pi_{N-1}^{z}\left(\operatorname{curl}_{k} \varphi\right)_{\theta} \\
\left(\operatorname{curl}_{k} \Pi_{N}^{c} \boldsymbol{\varphi}\right)_{z}=\tilde{\pi}_{N-1}^{1 r} \circ \pi_{N}^{1 z}\left(\operatorname{curl}_{k} \boldsymbol{\varphi}\right)_{z},
\end{gathered}
$$

where, for $m=1$ and 2 , the operator $\tilde{\pi}_{N}^{m r}$ is defined by

$$
\tilde{\pi}_{N}^{m r} \varphi=r^{-1} \pi_{N}^{m r}(r \varphi) .
$$


Let us recall from ([7], Sect. II.1), that the following estimates hold for all functions $\varphi$ in $H^{s}(-1,1), s \geq 0$,

$$
\left\|\varphi-\pi_{N-1}^{z} \varphi\right\|_{L^{2}(-1,1)} \leq c N^{-s}\|\varphi\|_{H^{s}(-1,1)},
$$

and for all functions $\varphi$ in $H^{s}(-1,1), s \geq 1$,

$$
\left\|\varphi-\pi_{N}^{1 z} \varphi\right\|_{L^{2}(-1,1)} \leq c N^{-s}\|\varphi\|_{H^{s}(-1,1)} .
$$

Similarly the following estimates hold for $m=1,2$ and for all functions $\varphi$ in $H_{1}^{s}(0,1), s \geq m+1$,

$$
\left\|\varphi-\pi_{N}^{m r} \varphi\right\|_{L^{2}(0,1)} \leq c N^{-s}\|\varphi\|_{H_{1}^{s}(0,1)} .
$$

Establishing an estimate for the operator $\tilde{\pi}_{N}^{m r}$ requires a further argument which is analogous to ([7], Thm. III.1.19, see the proof of this theorem).

Lemma B.1. The following estimate holds for all functions $\varphi$ in $H_{1}^{s}(0,1), s>m$,

$$
\left\|\varphi-\tilde{\pi}_{N}^{m r} \varphi\right\|_{L_{1}^{2}(0,1)} \leq c N^{-s}\|\varphi\|_{H_{1}^{s}(0,1)} .
$$

Proof. We only give the proof in the case $m=1$ since its analogue in the case $m=2$ is rather similar but more technical. Let us recall from ([7], Déf. II.1.8) that, for any function $\psi$ in $H^{1}(0,1)$, we have

$$
\psi-\pi_{N}^{1 r} \psi=\psi^{0}-\pi_{N}^{1 r} \psi^{0}, \quad \text { with } \quad \psi^{0}=\psi-(1-r) \psi(0)-r \psi(1),
$$

and the restriction of $\pi_{N}^{1 r}$ to $H_{0}^{1}(0,1)$ is nothing but the orthogonal projection from $H_{0}^{1}(0,1)$ onto $\mathbb{P}_{N}^{0}(0,1)$. Denoting by $\tilde{L}_{n}, n \geq 0$, the Legendre polynomials $L_{n}$ composed with the homotethy that maps $[0,1]$ onto $[-1,1]$, we write for any $\psi^{0}$ in $H_{0}^{1}(0,1)$

$$
\psi^{0}=\sum_{n=1}^{\infty} \alpha_{n} r(1-r) \tilde{L}_{n}^{\prime}, \quad \text { thus } \quad \pi_{N}^{1 r} \psi^{0}=\sum_{n=1}^{N-1} \alpha_{n} r(1-r) \tilde{L}_{n}^{\prime},
$$

so that

This yields

$$
\int_{0}^{1}\left(\psi^{0}-\pi_{N}^{1 r} \psi^{0}\right)^{2} r^{-1} \mathrm{~d} r \leq \int_{0}^{1}\left(\psi^{0}-\pi_{N}^{1 r} \psi^{0}\right)^{2} r^{-1}(1-r)^{-1} \mathrm{~d} r \leq c \sum_{n=N}^{\infty} \alpha_{n}^{2} n .
$$

$$
\int_{0}^{1}\left(\psi^{0}-\pi_{N}^{1 r} \psi^{0}\right)^{2} r^{-1} \mathrm{~d} r \leq c N^{-2}\left|\psi^{0}-\pi_{N}^{1 r} \psi^{0}\right|_{H^{1}(0,1)}^{2}
$$

Thus, we derive from (B.1) and (B.7) that, for all $s \geq 2$,

$$
\left\|\left(\psi^{0}-\pi_{N}^{1 r} \psi^{0}\right) r^{-1}\right\|_{L_{1}^{2}(0,1)} \leq c N^{-s}\left\|\psi^{0}\right\|_{H_{1}^{s}(0,1)} .
$$

The same estimate holds with $\psi^{0}$ replaced by $\psi$ (note that $H_{1}^{s}(0,1)$ is embedded in $\mathscr{C}^{0}([0,1])$ for $s \geq 1$ ), and of course with $\psi$ replaced by $r \varphi$, whence the desired result for $s \geq 2$. Moreover the previous estimate holds with $\left\|\psi^{0}\right\|_{H_{1}^{s}(0,1)}$ replaced by $\left\|\psi^{0}\right\|_{H^{s}(0,1)}$ for $s \geq 1$, see (B.6), and, from the previous embedding, $\psi^{0}$ can be replaced by $\psi$ for $s>1$. Finally, an inequality of Hardy's type gives

$$
\|r \varphi\|_{H^{s}(0,1)} \leq\|\varphi\|_{H_{1}^{s}(0,1)}
$$

for $s=1$ and $s=2$, whence for $1<s<2$ by an interpolation argument, which concludes the proof. 
Note that, in (B.3), we can write equivalently

$$
\left(\Pi_{N}^{c} \varphi\right)_{\theta}=\tilde{\pi}_{N}^{2 r} \circ \pi_{N}^{1 z} \varphi_{\theta} .
$$

So estimates for the operator $\Pi_{N}^{c}$ can be derived from (B.5) to (B.8) thanks to a tensorization argument.

Lemma B.2. The following estimate holds for all functions $\varphi$ in $H\left(\operatorname{curl}_{k}, \Omega\right) \cap H_{1}^{s}(\Omega)^{3}, s>3$,

$$
\left\|\varphi-\Pi_{N}^{c} \varphi\right\|_{L_{1}^{2}(\Omega)^{3}} \leq c N^{-s}\|\varphi\|_{H_{1}^{s}(\Omega)^{3}} .
$$

The following estimate holds for all functions $\varphi$ in $H\left(\operatorname{curl}_{k}, \Omega\right)$ such that $\operatorname{curl}_{k} \varphi$ belongs to $H_{1}^{s}(\Omega)^{3}, s>2$,

$$
\left\|\operatorname{curl}_{k}\left(\varphi-\Pi_{N}^{c} \varphi\right)\right\|_{L_{1}^{2}(\Omega)^{3}} \leq c N^{-s}\left\|\operatorname{curl}_{k} \varphi\right\|_{H_{1}^{s}(\Omega)^{3}} .
$$

Proof. For instance, we have the formula

$$
\left\|\varphi_{r}-\left(\Pi_{N}^{c} \varphi\right)_{r}\right\|_{L_{1}^{2}(\Omega)} \leq\left\|\varphi_{r}-\pi_{N-1}^{1 r} \varphi_{r}\right\|_{L_{1}^{2}(\Omega)}+\left\|\varphi_{r}-\pi_{N}^{1 z} \varphi_{r}\right\|_{L_{1}^{2}(\Omega)}+\left\|\left(i d-\pi_{N-1}^{1 r}\right)\left(\varphi_{r}-\pi_{N}^{1 z} \varphi_{r}\right)\right\|_{L_{1}^{2}(\Omega)},
$$

so that the estimate for $\left\|\varphi_{r}-\left(\Pi_{N}^{c} \varphi\right)_{r}\right\|_{L_{1}^{2}(\Omega)}$ follows from (B.6) and (B.7). Analogous arguments can be used for the other five terms.

\section{Approximation of any functions}

We now wish to extend the results of Lemma B.2 to less smooth functions. We first work with functions with zero tangential trace.

Lemma B.3. The following estimate holds for any function $\varphi$ in $X_{(k)}(\Omega)$ such that $\operatorname{curl}_{k} \varphi$ belongs to $H_{1}^{s}(\Omega)^{3}$, $s \geq 0$

$$
\inf _{\varphi_{N} \in \mathbb{X}_{N(k)}}\left\|\operatorname{curl}_{k}\left(\varphi-\varphi_{N}\right)\right\|_{L_{1}^{2}(\Omega)^{3}} \leq c N^{-s}\left\|\operatorname{curl}_{k} \varphi\right\|_{H_{1}^{s}(\Omega)^{3}} .
$$

Proof. We first observe from ([5], Thm. 3.17) that the mapping: $\boldsymbol{\varphi} \mapsto \operatorname{curl}_{k} \boldsymbol{\varphi}$ is continuous from $X_{(k)}(\Omega)$ onto the space

$$
Z_{(k)}(\Omega)=\left\{\boldsymbol{v} \in L_{1}^{2}(\Omega)^{3} ; \operatorname{div}_{k} \boldsymbol{v}=0 \text { in } \Omega \text { and } \gamma_{N} \boldsymbol{v}=0 \text { sur } \Gamma\right\},
$$

where the normal trace operator $\gamma_{N}$ is simply defined by $\gamma_{N} \boldsymbol{v}=v_{r} n_{r}+v_{z} n_{z}$. Let now $\tilde{\Pi}_{N}$ be the orthogonal projection operator from $Z_{(k)}(\Omega)$ onto the range $\operatorname{curl}_{k} \mathbb{X}_{N(k)}$ of the space $\mathbb{X}_{N(k)}$ by the operator $\operatorname{curl}_{k}$ associated with the scalar product of $L_{1}^{2}(\Omega)^{3}$. Then, the estimate

$$
\left\|\boldsymbol{v}-\tilde{\Pi}_{N} \boldsymbol{v}\right\|_{L_{1}^{2}(\Omega)^{3}} \leq c N^{-s}\|\boldsymbol{v}\|_{H_{1}^{s}(\Omega)^{3}},
$$

holds for $s=0$ by definition and for $s>2$ from (B.10). So the desired result follows by an interpolation argument between the spaces $Z_{(k)}(\Omega) \cap H_{1}^{s}(\Omega)^{3}, s=0$ and $s>2$, which can be derived from an extension of ([9], Rem. 4.13) to the case of a cylinder.

The same argument can be used for functions in $Y_{(k)}(\Omega)$, but this does not lead to estimate of $\left\|\varphi-\varphi_{N}\right\|_{L_{1}^{2}(\Omega)^{3}}$ (the corresponding interpolation property seems presently unknown). So to conclude the proof of Lemma 4.12 we are led to use the orthogonal projection operator from $\mathbf{H}_{(k)}^{1}(\Omega)$ onto $\mathbb{Y}_{N(k)}$. The next result is derived from ([10], Sect. V.3).

Lemma B.4. The following estimate holds for any function $\varphi$ in $Y_{(k)}(\Omega) \cap H_{1}^{s}(\Omega)^{3}, s>1$,

$$
\inf _{\varphi_{N} \in \mathbb{Y}_{N(k)}}\left(N\left\|\boldsymbol{\varphi}-\boldsymbol{\varphi}_{N}\right\|_{L_{1}^{2}(\Omega)^{3}}+\left\|\operatorname{curl}_{k}\left(\boldsymbol{\varphi}-\boldsymbol{\varphi}_{N}\right)\right\|_{L_{1}^{2}(\Omega)^{3}}\right) \leq c N^{1-s}\|\varphi\|_{H_{1}^{s}(\Omega)^{3}} .
$$




\section{A further result}

In order to prove Lemma 4.13, we introduce the modified operator $\tilde{\Pi}_{N}^{c}$, defined as follows on smooth enough vector fields $\varphi$ :

$$
\left(\tilde{\Pi}_{N}^{c} \varphi\right)_{r}=\pi_{N-1}^{2 r} \circ \pi_{N}^{2 z} \varphi_{r}, \quad\left(\tilde{\Pi}_{N}^{c} \varphi\right)_{\theta}=r^{-1} \pi_{N}^{2 r} \circ \pi_{N}^{2 z}\left(r \varphi_{\theta}\right), \quad\left(\tilde{\Pi}_{N}^{c} \varphi\right)_{z}=\pi_{N}^{2 r} \circ \pi_{N-1}^{1 z} \varphi_{z}
$$

where the operator $\pi_{N}^{2 z}$ is the analogue on ]-1, 1 [ of the operator $\pi_{N}^{2 r}$. This operator has the same properties as $\Pi_{N}^{c}$, plus the further one: if $\gamma_{T}\left(\operatorname{curl}_{k} \boldsymbol{\varphi}\right)$ is equal to zero, so is $\gamma_{T}\left(\operatorname{curl}_{k} \tilde{\Pi}_{N}^{c} \boldsymbol{\varphi}\right)$. Let $\tilde{\mathbb{X}}_{N(k)}$ be the subspace of vector fields $\boldsymbol{\varphi}_{N}$ in $\mathbb{X}_{N(k)}$ such that $\gamma_{T}\left(\operatorname{curl}_{k} \boldsymbol{\varphi}_{N}\right)$ is equal to zero.

Lemma B.5. The following estimate holds for any function $\varphi$ in $X_{(k)}(\Omega)$ such that $\operatorname{curl}_{k} \varphi$ belongs to $H_{1}^{t}(\Omega)^{3}$, $t \geq 1$, and $\gamma_{T}\left(\operatorname{curl}_{k} \boldsymbol{\varphi}\right)$ is equal to zero,

$$
\inf _{\boldsymbol{\varphi}_{N} \in \tilde{\mathbb{X}}_{N(k)}}\left\|\operatorname{curl}_{k} \operatorname{curl}_{k}\left(\boldsymbol{\varphi}-\boldsymbol{\varphi}_{N}\right)\right\|_{L_{1}^{2}(\Omega)^{3}} \leq c N^{\frac{3}{2}-t}\left\|\operatorname{curl}_{k} \boldsymbol{\varphi}\right\|_{H_{1}^{t}(\Omega)^{3}}
$$

Proof. The same arguments as for (B.10) and a further investigation of the property of the operator $\tilde{\pi}_{N}^{1 r}$ in the $H_{1}^{1}(0,1)$-seminorm yields that (B.14) holds with $\varphi_{N}=\tilde{\Pi}_{N}^{c} \varphi$ for $t>\frac{7}{2}$. So, we conclude by the same arguments as for Lemma B.3, by noting that, for any function $\varphi$ satisfying the assumptions of the lemma, $\operatorname{curl}_{k} \varphi$ belongs to

$$
\tilde{Z}_{(k)}(\Omega)=\left\{\boldsymbol{v} \in H_{1 \diamond}^{1}(\Omega)^{3} ; \operatorname{div}_{k} \boldsymbol{v}=0 \text { in } \Omega\right\}
$$

and using an interpolation argument between the spaces $\tilde{Z}_{(k)}(\Omega) \cap H_{1}^{t}(\Omega)^{3}$ relying on ([9], Cor. 4.6).

\section{REFERENCES}

[1] N. Abdellatif, Méthodes spectrales et d'éléments spectraux pour les équations de Navier-Stokes axisymétriques. Thesis, Université Pierre et Marie Curie, Paris (1997).

[2] N. Abdellatif, A mixed stream function and vorticity formulation for axisymmetric Navier-Stokes equations. J. Comp. Appl. Math. 117 (2000) 61-83.

[3] M. Amara, H. Barucq and M. Duloué, Une formulation mixte convergente pour le système de Stokes tridimensionnel. $C$. $R$. Acad. Sci. Paris Série I 328 (1999) 935-938.

[4] M. Amara, H. Barucq and M. Duloué, Une formulation mixte convergente pour les équations de Stokes tridimensionnelles. Actes des VI ${ }^{\mathrm{es}}$ Journées Zaragoza-Pau de Mathématiques Appliquées et de Statistiques, Publ. Univ. Pau, Pau (2001) 61-68.

[5] C. Amrouche, C. Bernardi, M. Dauge and V. Girault, Vector potentials in three-dimensional nonsmooth domains. Math. Meth. Appl. Sci. 21 (1998) 823-864.

[6] F. Ben Belgacem and C. Bernardi, Spectral element discretization of the Maxwell equations. Math. Comput. 68 (1999) $1497-1520$.

[7] C. Bernardi and Y. Maday, Approximations spectrales de problèmes aux limites elliptiques. Springer-Verlag. Math. Appl. 10 (1992).

[8] C. Bernardi, V. Girault and Y. Maday, Mixed spectral element approximation of the Navier-Stokes equations in the streamfunction and vorticity formulation. IMA J. Numer. Anal. 12 (1992) 565-608.

[9] C. Bernardi, M. Dauge and Y. Maday, Interpolation of nullspaces for polynomial approximation of divergence-free functions in a cube, in Proc. Conf. Boundary Value Problems and Integral Equations in Non smooth Domains, M. Costabel, M. Dauge and S. Nicaise Eds., Dekker. Lect. Notes Pure Appl. Math. 167 (1994) 27-46.

[10] C. Bernardi, M. Dauge, Y. Maday and M. Azaïez, Spectral Methods for Axisymmetric Domains. Gauthier-Villars \& NorthHolland. Ser. Appl. Math. 3 (1999).

[11] C. Canuto and A. Quarteroni, Approximation results for orthogonal polynomials in Sobolev spaces. Math. Comput. 38 (1982) $67-86$.

[12] M. Costabel and M. Dauge, Singularities of electromagnetic fields in polyhedral domains. Arch. Ration. Mech. Anal. 151 (2000) 221-276.

[13] M. Duloué, Analyse numérique des problèmes d'écoulement de fluides. Thesis, Université de Pau et des Pays de l'Adour, Pau (2001). 
[14] V. Girault and P.-A. Raviart, An analysis of a mixed finite element method for the Navier-Stokes equations. Numer. Math. 33 (1979) 235-271.

[15] V. Girault and P.-A. Raviart, Finite Element Methods for the Navier-Stokes Equations, Theory and Algorithms. SpringerVerlag (1986).

[16] R. Glowinski and O. Pironneau, Numerical methods for the first biharmonic equation and for the two-dimensional Stokes problem. SIAM Review 21 (1979) 167-212.

To access this journal online: www.edpsciences.org 